\title{
A comprehensive survey on Cultural Algorithms
}

\author{
Alireza Maheri ${ }^{a}$, Shahin Jalilii, ${ }^{*}$, Yousef Hosseinzadeh ${ }^{b}$, Reza Khani $^{b}$, Mirreza \\ Miryahyavi $^{b}$ \\ ${ }^{a}$ School of Engineering, University of Aberdeen, King's College, Aberdeen, AB24 3UE, \\ United Kingdom \\ ${ }^{b}$ Faculty of Civil Engineering, University of Tabriz, Tabriz, Iran
}

\section{Abstract}

Cultural Algorithms (CAs) are evolutionary algorithms (EAs) inspired by the conceptual models of the human cultural evolution process. In contrast to the conventional EAs, which work only based on the population space, CAs employ an additional space, called belief space, to collect the information about the behaviour of individuals within the search space. Since the emergence of CAs, they have been extended to solve a wide variety of problems in different branches of science and technology. In this paper, a comprehensive survey on the recent advances in CAs is presented. Literature survey reveals some interesting challenges and future research directions.

\section{Keywords}

Cultural algorithms, evolutionary algorithms, belief space, cultural evolution.

\section{Nomenclature}
ABC Artificial Bee Colony
ACO Ant Colony Optimisation
ANN Artificial Neural Network

BA Bat Algorithm

BB-BC Big Bang-Big Crunch

BBO Biogeography-Based Optimisation

BFA Bacterial Foraging Algorithm

CAs Cultural Algorithms

CBO Colliding Bodies Optimisation

COA Coyote Optimisation Algorithm

\footnotetext{
*Corresponding author: Shahin Jalili, email: s.jalili@abdn.ac.uk, School of Engineering, University of Aberdeen, King's College, Aberdeen, AB24 3UE, United Kingdom
} 
CPSO Cooperative Particle Swarm Optimisation

CS Cuckoo Search

CSS Charged System Search

DE Differential Evolution

EAs Evolutionary algorithms

EDP Economic Dispatch Problem

EHO Elephant herding optimisation

EP Evolutionary Programming

FA Firefly Algorithm

FECO Five-Elements Cycle Optimisation

GAs Genetic Algorithms

GP Genetic Programming

GSA Gravitational Search Algorithm

GSO Group Search Optimiser

HICHA Heterogeneous Interactive Cultural Hybrid Algorithm

HS Harmony Search

IQPSO Improved Quantum-behaved Particle Swarm Optimisation

IWO Invasive Weed Optimisation

JSP Job Shop Problem

$\mathrm{KH} \quad$ Krill Herd

LCA League Championship Algorithm

MODE Multi-objective Differential Evolution

NSGA-II Non-dominating Sorting Genetic Algorithm II

OIO Optics Inspired Optimisation

PSO Particle Swarm Optimisation

QEA Quantum Evolution Algorithm

rcGA Real-Coded Genetic Algorithm

SA Simulated Annealing

SFLA Shuffled Frog Leaping Algorithm

SI Swarm Intelligence

SOMA Self-Organising Migrating Algorithm

TLBO Teaching-Learning-Based Optimisation

TS Tabu Search 


\section{Contents}

1 Introduction 4

2 Cultural Algorithms (CAs) $\quad 6$

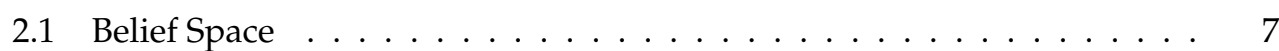

2.1.1 Situational knowledge . . . . . . . . . . . . . . . . . . 10

2.1.2 Normative knowledge . . . . . . . . . . . . . . . . . . . . . 10

2.1.3 Topographical knowledge . . . . . . . . . . . . . . . . . . . 11

2.1 .4 Domain knowledge . . . . . . . . . . . . . . . . . 12

2.1 .5 History knowledge . . . . . . . . . . . . . . . . . . . 12

2.2 Communication Protocol . . . . . . . . . . . . . . . . . . . 12

2.2 .1 Acceptance function . . . . . . . . . . . . . . 13

2.2.2 Influence function $\ldots \ldots \ldots \ldots \ldots$

2.3 Algorithmic steps of CAs . . . . . . . . . . . . . . . . . . . 14

3 Applications of CAs $\quad 15$

3.1 Civil engineering . . . . . . . . . . . . . . . . . 18

3.2 Mechanical engineering . . . . . . . . . . . . . . . . . . . 19

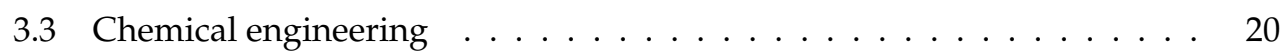

3.4 Electrical engineering . . . . . . . . . . . . . . . . . . . 21

3.5 Computer science . . . . . . . . . . . . . . . . . . . . . . . . 24

3.5 .1 Function optimisation $\ldots \ldots \ldots \ldots \ldots$

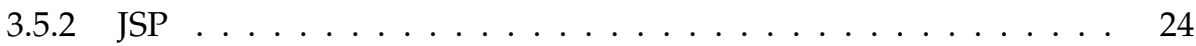

3.5 .3 Facility location . . . . . . . . . . . . . . 25

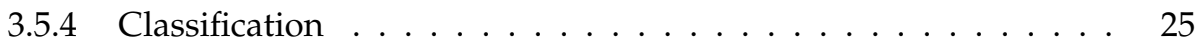

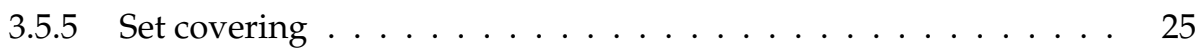

3.5 .6 Others . . . . . . . . . . . . . . . . . . 25

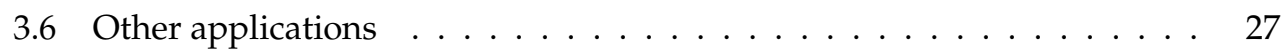

4 Variants of CAs $\quad 28$

4.1 Hybridisation of CAs . . . . . . . . . . . . . . . 28

$4.1 .1 \quad$ Hybrid CA and GA . . . . . . . . . . . . . . . . . 29

4.1 .2 Hybrid CA and PSO . . . . . . . . . . . . . . . . . . 29

4.1 .3 Hybrid CA and DE . . . . . . . . . . . . . . . 32 
4.1.4 Hybridisation with local search . . . . . . . . . . . . 33

4.1.5 Other hybridisation schemes .................. 33

4.2 Communication topology for CAs . . . . . . . . . . . . 35

4.3 Improved CA . . . . . . . . . . . . . . 36

4.4 Multi-objective CA . . . . . . . . . . . . . . . 37

4.5 Multi-population CA . . . . . . . . . . . . . 38

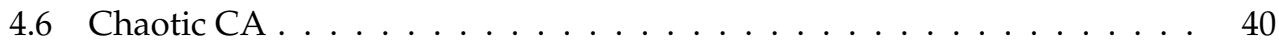

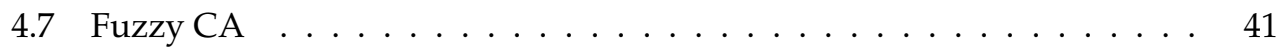

4.8 CAs with EP and GP . . . . . . . . . . . . . . . . 41

5 Publication analysis $\quad 41$

6 Conclusions and future research directions $\quad 43$

$\begin{array}{ll}\text { References } & 44\end{array}$

\section{Introduction}

With rapid development of science and technology as well as emergence of complex real-world optimisation problems, developing efficient solution-finding approaches has become a research hotspot in the computer science. In most of the practical optimisation problems, due to the presence of highly non-linear objective and constraints functions with mixed types of continuous/discrete variables, finding acceptable solutions is not an easy task. Another source of difficulty stems from the fact that most of the real-world optimisation problems are black-box problems, in which the explicit forms of the objective and constraints functions as well as their derivatives are not available. These have motivated the development of meta-heuristic optimisation techniques to provide optimal/near-optimal solutions for these types of problems in a reasonable time.

Significant progress has been made over the past decades in mimicking the physical, ecological and social phenomena to develop meta-heuristic algorithms as efficient tools of finding acceptable solutions for complex real-world optimisation problems (Del Ser et al., 2019), such as GAs (Mühlenbein, 1997), DE (Storn, Price, 1997), PSO (Eberhart, Kennedy, 1995), HS (Geem et al., 2001), ACO (Dorigo, Di Caro, 1999), (CAs) (Reynolds, 1994), TLBO (Rao et al., 2011), ABC (Karaboga et al., 2014), BBO (Simon, 2008), BB-BC (Erol, Eksin, 2006), LCA (Kashan, 2009; Jalili et al., 2017), CSS (Kaveh, Talatahari, 2010), OIO (Jalili, Husseinzadeh Kashan, 2019), CBO (Kaveh, Mahdavi, 2014), GSO (He et al., 2009), GSA (Rashedi et al., 2009), and KH (Gandomi, Alavi, 2012) algorithm. In contrast to the conventional gradient-based optimisation techniques, the meta-heuristic techniques can be easily implemented to deal with the optimisation problems with different characteristics, such as non-linearity, non-separability, illconditioning, and multi-modality (Ali et al., 2018).

Meta-heuristic techniques can be categorised into different classes based on their 
source of inspiration, such as EAs, SI algorithms, and Physics inspired algorithms. EAs are biologically inspired meta-heuristic algorithms based on the Darwinian theory of evolution, in which the survival capabilities of biological species are improved through the natural processes such as selection, reproduction, and survival of the fittest. For example, GAs, DE, and BBO belong to the EAs category. SIs mimic the collective intelligence exhibited by a group or swarm of species in the nature, such as birds, ants, fishes, etc. PSO, ABC algorithm, and ACO are examples of SI methods, which are developed based on the swarming behaviour of a flock of birds and colony of ants. As its name suggests, Physics inspired algorithms simulate the physical processes in the nature through their governing theories and equations to design a search mechanism. For example, CSS simulates the Newtonian laws of classical mechanics and Coulomb law between the charged particles to perform the search process for global optima, OIO method imitates the law of reflection and the optical behaviour of convex and concave mirrors to design a search mechanism for numerical optimisation, GSA performs based on the simulation of the law of gravity and the interactions between the masses, CBO mimics the one dimensional collision process of bodies based on the laws of energy and momentum. The overall framework of the above mentioned meta-heuristic approaches is more or less the same and they usually are referred to as the population-based metaheuristics, in which a population of individual solutions are gradually updated within the search process. Some of the meta-heuristic algorithms, such as SA (Van Laarhoven, Aarts, 1987) and TS (Glover, Laguna, 1998), perform based on the updating of a single solution, which are referred to as single-point or trajectory search methods.

The idea of using multiple sources of knowledge during the search process is emerged with the development of CAs. CAs developed by Reynolds (1994) can be categorised as an EA and is based on the conceptual models of the human cultural evolution process. In contrast to the conventional EAs, which work only based on the population space, CAs employ an additional space, called belief space, to collect the information about the behaviour of individuals in the search space (Reynolds, 2018a,b; Chung, Reynolds, 1996a; Reynolds, Kinnaird-Heether, 2017a; Reynolds, Maletic, 1994; Saleem, Reynolds, 2000; Reynolds et al., 2008c; Reynolds, 1999). CAs model a dual inheritance system observed from the human culture evolution, in which the belief space represents the macro-evolutionary level and the population space performs the micro-evolutionary level. The belief space includes a network of knowledge categories obtained from the evolution process of individuals in the population space, in which each knowledge category indicates a collection of problem-specific knowledge components. The belief and population spaces can interact with each other during the evolution process. Experience exchange between these two spaces is performed based on the communication protocols. Since the emergence of fundamental CAs in 1979 (Reynolds, 1979), they have been successfully extended and widely employed to solve a wide variety of problems in different brunches of science and technology. Hence, a literature review of CAs seems to be crucial to provide a clear picture of their development trends and to highlight challenges and future research directions. The survey reported in this paper covers recent advances in CAs, their general framework, different variants, hybridised versions with other meta-heuristic and search techniques, and their applications in science and engineering.

The general structure of this paper is organised as follows. Section 2 provides the general framework and basic concept of CAs and their prominent variants and formulations. The literature review of CAs in relation to their applications in different 
branches of science and engineering is provided in Section 3. The scientific literature with respect to the hybridisation, multi-objective, multi-population, chaotic, fuzzy, and other variants of CAs are reviewed in Section 4. A brief publication analysis is discussed in Section 5. Finally, the concluding remarks and discussion on the future research in the field are presented in Section 6.

\section{Cultural Algorithms (CAs)}

According to Richerson, Boyd (2005), culture can be defined as a source of information with potential capability of affecting members of a population, which are exchanged between the individuals. This information can be any kind of mental state, such as the beliefs, art, and other things, which can be transmitted to others by means of teaching, imitation, and other forms of social transmission (Richerson, Boyd, 2005). Based on the biocultural evolution theory (Laland, 2008), genes and culture can be viewed as two interacting forms of inheritance. This means that the human behaviour is a product of two different and interacting evolutionary processes, including genetic evolution and cultural evolution. The variations in genes can lead to variations in the culture which can then influence genetic selection, and vice versa *. Reynolds (1979) inspired this concept to model new meta-heuristic optimisation algorithms, called CAs, based on the human cultural evolution process.

Conventional EAs have been indicated to be effective in solving different and complicated optimisation problems. However, the individual solutions exchange the limited amount of knowledge related to the problem between each other and the search process utilised by the standard EAs is unbiased (Engelbrecht, 2007). While the acquired knowledge during the evolution process can be stored and collectively used to perform search more efficiently (Reynolds, 2018a). CAs try to use a set of networks of knowledge sources gained during the evolution process to influence the search process. Fig. 1 shows the general framework and main components of CAs. In contrast to the conventional EAs, which are consisted of a population space, CAs work on two spaces, including population and belief spaces. In CAs, the learning process is performed in both of these spaces simultaneously. The interaction between the population and belief spaces is provided through the communication protocol, i.e., acceptance and influence functions. Like other population-based EAs, the population space of CAs is a genetic component containing individual solutions which are represented on a genotypic and/or phenotypic level. While the belief space represents the cultural information gained during the evolution process.

The general pseudo-code of CAs can be summarised as Algorithm 1. Like other meta-heuristic algorithms, the algorithm starts with a randomly generated population space represented by $P S(t)$. In addition, the general structure of the belief space indicated by $B S(t)$ is initialised by appropriate values. Then, the fitness functions of individuals in $P S(t)$ are evaluated using $\operatorname{Obj}()$ function. A given number of individuals with better fitness functions are selected by Accept() function from the $P S(t)$ to update the belief space $B S(t)$ using Update() function. Finally, the function Influence() is used to produce new generation of individuals $P S(t+1)$. The main components of CAs are explained in the following subsections.

*https://en.wikipedia.org/wiki/Dual ${ }_{i}$ nheritance $_{t}$ heory 


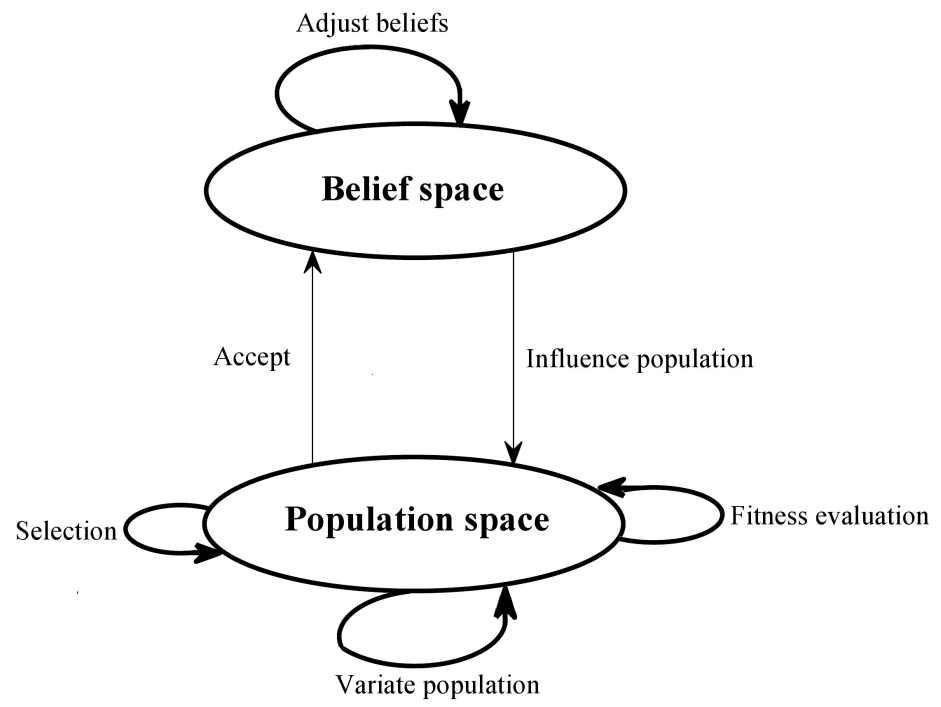

Figure 1: Overall framework of CA.

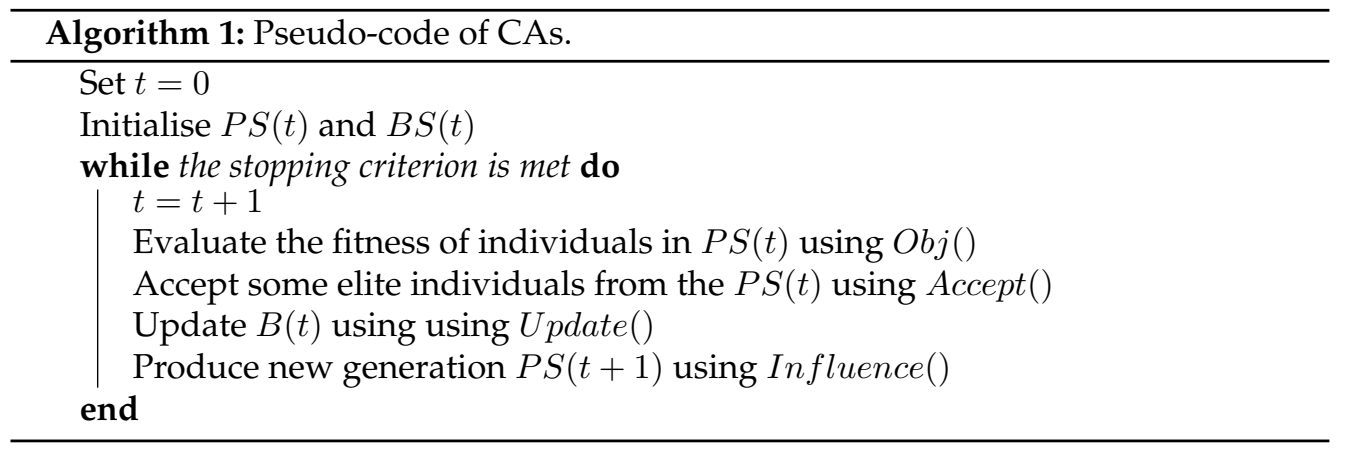

\subsection{Belief Space}

During the evolution process, the population space has a set of cultural information which are changing from generation to generation. In order to implement a learning mechanism, the belief space of CAs plays a role of cultural information repository, and stores the knowledge and collective behaviour acquired by the individuals. The primary version of CA was consisted of only one knowledge source (i.e., situational knowledge) (Reynolds, 1979) and it has been complemented by adding four additional knowledge sources, including normative, domain, history, and topographical knowledge sources. These knowledge and cultural information will be used as auxiliary tools to influence the future behaviour of the individuals and move them toward the global optima in the search space. The belief space forms a network of knowledge sources to provide an effective learning capability at different levels of granularity, which are gradually updated. The types of the applied knowledge sources within the belief space depend on the problem type. Literature review reveals that researchers have been used different knowledge sources within the belief space to solve different problems. Table 1 
lists the types of knowledge sources implemented by different studies within the belief space. As it is observed from Table 1, the normative and situational knowledge sources are popular knowledge components in literature.

Table 1: The knowledge sources of CA employed by different references in the literature.

\begin{tabular}{|c|c|c|c|c|c|}
\hline Reference & Situational & Normative & Domain & History & Topographical \\
\hline Haldar, Chakraborty (2014) & $\checkmark$ & & & $\checkmark$ & \\
\hline Haldar, Chakraborty (2015) & $\checkmark$ & & & $\checkmark$ & \\
\hline Arpaia et al. (2007) & $\checkmark$ & & & $\checkmark$ & $\checkmark$ \\
\hline Jalili, Hosseinzadeh (2015) & $\checkmark$ & $\checkmark$ & & & \\
\hline Jalili et al. (2019) & $\checkmark$ & $\checkmark$ & & & \\
\hline Jafari et al. (2019) & $\checkmark$ & $\checkmark$ & & & \\
\hline Ali et al. (2018) & $\checkmark$ & $\checkmark$ & $\checkmark$ & & \\
\hline Yan et al. (2017a) & $\checkmark$ & $\checkmark$ & & & \\
\hline Liu, Lin (2015) & $\checkmark$ & $\checkmark$ & & & \\
\hline Guedria, Hassine (2018) & $\checkmark$ & $\checkmark$ & & & \\
\hline Ali, Reynolds (2014) & $\checkmark$ & $\checkmark$ & $\checkmark$ & $\checkmark$ & $\checkmark$ \\
\hline Chen et al. (2008) & & $\checkmark$ & & & \\
\hline Wang et al. (2015) & $\checkmark$ & $\checkmark$ & & & \\
\hline Haikal, El-Hosseni (2011) & $\checkmark$ & & & $\checkmark$ & \\
\hline Xu et al. (2010) & $\checkmark$ & $\checkmark$ & & & \\
\hline Xu et al. (2012) & $\checkmark$ & $\checkmark$ & & & \\
\hline Tremayne et al. (2009) & $\checkmark$ & $\checkmark$ & & $\checkmark$ & $\checkmark$ \\
\hline $\mathrm{Gu}, \mathrm{Wu}(2010)$ & $\checkmark$ & & & & \\
\hline Gao et al. (2019) & $\checkmark$ & $\checkmark$ & & & \\
\hline Chen et al. (2013) & $\checkmark$ & & & & \\
\hline Ya-Li, Li-qing (2010) & $\checkmark$ & $\checkmark$ & & & \\
\hline Goudarzi et al. (2017) & $\checkmark$ & $\checkmark$ & & & \\
\hline Goudarzi et al. (2016) & $\checkmark$ & $\checkmark$ & & & \\
\hline Bhattacharya et al. (2012a) & & $\checkmark$ & & & \\
\hline Ali et al. (2016b) & $\checkmark$ & $\checkmark$ & & $\checkmark$ & $\checkmark$ \\
\hline Wu et al. (2010) & $\checkmark$ & $\checkmark$ & & & \\
\hline Stanley et al. (2019) & $\checkmark$ & $\checkmark$ & $\checkmark$ & $\checkmark$ & $\checkmark$ \\
\hline Awad et al. (2013) & $\checkmark$ & $\checkmark$ & $\checkmark$ & $\checkmark$ & $\checkmark$ \\
\hline $\begin{array}{l}\text { Abdolrazzagh-Nezhad } \\
(2020)\end{array}$ & $\checkmark$ & $\checkmark$ & & & \\
\hline Lin et al. (2009) & & $\checkmark$ & & & \\
\hline Oloruntoba et al. (2019) & $\checkmark$ & $\checkmark$ & & & \\
\hline Sun et al. (2009) & $\checkmark$ & $\checkmark$ & & & \\
\hline $\begin{array}{l}\text { Mohammadhosseini et al. } \\
\text { (2019) }\end{array}$ & $\checkmark$ & $\checkmark$ & & & \\
\hline Coello Coello, Becerra (2004) & $\checkmark$ & $\checkmark$ & & & \\
\hline Chung, Reynolds (1996b) & $\checkmark$ & $\checkmark$ & & & \\
\hline $\begin{array}{l}\text { Santos Coelho dos et al. } \\
\text { (2009) }\end{array}$ & $\checkmark$ & $\checkmark$ & & & \\
\hline De Freitas et al. (2018) & $\checkmark$ & $\checkmark$ & & & $\checkmark$ \\
\hline Khan et al. (2014) & $\checkmark$ & $\checkmark$ & & & \\
\hline Khan et al. (2017) & $\checkmark$ & $\checkmark$ & & & \\
\hline Guo et al. (2013b) & $\checkmark$ & $\checkmark$ & & & \\
\hline Guo et al. (2013a) & $\checkmark$ & $\checkmark$ & & & \\
\hline Gao, Diao (2011) & $\checkmark$ & $\checkmark$ & & & \\
\hline Gao, Xu (2013) & & $\checkmark$ & & & \\
\hline Digalakis, Margaritis (2002) & $\checkmark$ & $\checkmark$ & & & \\
\hline Gao et al. (2017a) & $\checkmark$ & $\checkmark$ & & & \\
\hline Khodabakhshian, Hemmati & $\checkmark$ & $\checkmark$ & & & \\
\hline
\end{tabular}


Table 1 - Continued from previous page

\begin{tabular}{|c|c|c|c|c|c|}
\hline Reference & Situational & Normative & Domain & History & Topographical \\
\hline Bhattacharya et al. (2012b) & $\checkmark$ & $\checkmark$ & & & \\
\hline Lu et al. (2011) & $\checkmark$ & & & $\checkmark$ & \\
\hline Yuan et al. (2009) & $\checkmark$ & $\checkmark$ & & & \\
\hline Yuan et al. (2008) & $\checkmark$ & $\checkmark$ & & & \\
\hline Cai et al. (2018) & $\checkmark$ & $\checkmark$ & & & $\checkmark$ \\
\hline $\begin{array}{l}\text { Santos Coelho dos, Mariani } \\
\text { (2006) }\end{array}$ & & $\checkmark$ & & & \\
\hline Xie et al. (2009) & $\checkmark$ & $\checkmark$ & & & \\
\hline Ali et al. (2014a) & $\checkmark$ & $\checkmark$ & $\checkmark$ & & $\checkmark$ \\
\hline Zhou et al. (2019) & $\checkmark$ & $\checkmark$ & & & \\
\hline Yan et al. (2017b) & & $\checkmark$ & & & \\
\hline Wang et al. (2017) & $\checkmark$ & $\checkmark$ & $\checkmark$ & & \\
\hline Pan et al. (2010) & & $\checkmark$ & & & \\
\hline Chung, Reynolds (1998) & $\checkmark$ & $\checkmark$ & & & \\
\hline Becerra, Coello (2004) & $\checkmark$ & $\checkmark$ & & $\checkmark$ & $\checkmark$ \\
\hline Pierezan et al. (2019) & & $\checkmark$ & & & \\
\hline Shah, Kobti (2020) & $\checkmark$ & $\checkmark$ & & & \\
\hline Mao et al. (2020) & $\checkmark$ & $\checkmark$ & & & $\checkmark$ \\
\hline Goli et al. (2019) & $\checkmark$ & $\checkmark$ & & & \\
\hline Mojab et al. (2019) & $\checkmark$ & $\checkmark$ & $\checkmark$ & & \\
\hline $\begin{array}{l}\text { Murugadass, Sivakumar } \\
(2020)\end{array}$ & $\checkmark$ & $\checkmark$ & & & \\
\hline Xue $(2020)$ & $\checkmark$ & $\checkmark$ & & & \\
\hline Muhamediyeva (2020) & $\checkmark$ & $\checkmark$ & & & \\
\hline Amalo et al. (2020) & & $\checkmark$ & & & \\
\hline Selvarajah et al. (2020) & & $\checkmark$ & & & $\checkmark$ \\
\hline Xue et al. (2011) & $\checkmark$ & $\checkmark$ & & & \\
\hline Guo et al. (2011b) & & $\checkmark$ & & & $\checkmark$ \\
\hline Gao et al. (2006a) & & $\checkmark$ & & & \\
\hline Gao, Diao (2010) & $\checkmark$ & $\checkmark$ & & & \\
\hline Gao et al. (2007) & $\checkmark$ & & & & \\
\hline Nguyen, Yao (2006) & $\checkmark$ & $\checkmark$ & & & \\
\hline Ali et al. (2011a) & $\checkmark$ & $\checkmark$ & $\checkmark$ & $\checkmark$ & $\checkmark$ \\
\hline Guo et al. (2018) & $\checkmark$ & $\checkmark$ & & & $\checkmark$ \\
\hline Kim, Cho (2009) & $\checkmark$ & $\checkmark$ & & $\checkmark$ & $\checkmark$ \\
\hline Guo et al. (2011c) & $\checkmark$ & $\checkmark$ & & & \\
\hline Reynolds, Zhu (2001) & $\checkmark$ & $\checkmark$ & & & \\
\hline $\mathrm{He}, \mathrm{Xu}(2011)$ & $\checkmark$ & $\checkmark$ & & & \\
\hline Sun et al. (2010) & $\checkmark$ & $\checkmark$ & & & \\
\hline Awad et al. (2017) & $\checkmark$ & $\checkmark$ & $\checkmark$ & & $\checkmark$ \\
\hline Ali et al. (2016a) & $\checkmark$ & $\checkmark$ & & & $\checkmark$ \\
\hline Ali, Awad (2014) & $\checkmark$ & $\checkmark$ & $\checkmark$ & $\checkmark$ & $\checkmark$ \\
\hline Ali et al. (2012) & $\checkmark$ & $\checkmark$ & $\checkmark$ & $\checkmark$ & $\checkmark$ \\
\hline Cortés Rivera et al. (2007) & $\checkmark$ & & & & \\
\hline Zhang, Zhu (2012) & $\checkmark$ & $\checkmark$ & & & \\
\hline Yang, Gu (2014) & $\checkmark$ & $\checkmark$ & & & $\checkmark$ \\
\hline Becerra, Coello (2005) & $\checkmark$ & & & & \\
\hline Alami et al. (2007) & & $\checkmark$ & & & \\
\hline Lagos et al. (2014) & $\checkmark$ & $\checkmark$ & & $\checkmark$ & \\
\hline Cabrera et al. (2011) & $\checkmark$ & & & $\checkmark$ & \\
\hline $\begin{array}{l}\text { Srinivasan, Ramakrishnan } \\
\text { (2012) }\end{array}$ & $\checkmark$ & $\checkmark$ & $\checkmark$ & $\checkmark$ & $\checkmark$ \\
\hline Crawford et al. (2013) & $\checkmark$ & & & $\checkmark$ & \\
\hline Crawford et al. (2007) & $\checkmark$ & & & & \\
\hline Soza et al. (2011) & $\checkmark$ & $\checkmark$ & $\checkmark$ & & \\
\hline Soza et al. (2007) & $\checkmark$ & $\checkmark$ & $\checkmark$ & & \\
\hline Zadeh, Kobti (2015) & $\checkmark$ & $\checkmark$ & & & \\
\hline
\end{tabular}


Table 1 - Continued from previous page

\begin{tabular}{llllll}
\hline Reference & Situational & Normative & Domain & History & Topographical \\
\hline Chen, Yang (2015) & $\checkmark$ & $\checkmark$ & $\checkmark$ & $\checkmark$ & $\checkmark$ \\
Terán et al. (2017) & $\checkmark$ & $\checkmark$ & & & \\
Santos Coelho dos, Alotto & & $\checkmark$ & & & \\
(2009) & & & & \\
Xue, Guo (2007) & $\checkmark$ & $\checkmark$ & & $\checkmark$ & $\checkmark$ \\
Ali et al. (2014b) & $\checkmark$ & $\checkmark$ & $\checkmark$ & $\checkmark$ & $\checkmark$ \\
Ali et al. (2011b) & $\checkmark$ & $\checkmark$ & $\checkmark$ & $\checkmark$ & $\checkmark$ \\
Best et al. (2010) & $\checkmark$ & $\checkmark$ & $\checkmark$ & $\checkmark$ & $\checkmark$ \\
Reynolds, Ali (2008b) & $\checkmark$ & $\checkmark$ & $\checkmark$ & $\checkmark$ & $\checkmark$ \\
Reynolds et al. (2008c) & $\checkmark$ & $\checkmark$ & $\checkmark$ & $\checkmark$ & $\checkmark$ \\
Reynolds, Peng (2005) & $\checkmark$ & $\checkmark$ & $\checkmark$ & $\checkmark$ & $\checkmark$ \\
\hline \multicolumn{7}{c}{ Continued on next page } \\
\end{tabular}

The belief space can be mathematically expressed as follows:

$$
\mathcal{B}^{t}=\left\{\mathcal{S}^{t}, \mathcal{N}^{t}, \mathcal{C}^{t}, \mathcal{D}^{t}, \mathcal{H}^{t}\right\}
$$

where $\mathcal{B}^{t}$ represents the belief space, $\mathcal{S}^{t}$ is situational knowledge source, $\mathcal{N}^{t}$ indicates the normative knowledge source, $\mathcal{C}^{t}$ is the topographical knowledge source, $\mathcal{D}^{t}$ is the domain knowledge source, and $\mathcal{H}^{t}$ represents the history knowledge source. The superscript $t$ indicates the iteration number. In the following subsections, each of the knowledge sources are explained in detail.

\subsubsection{Situational knowledge}

The situational knowledge source represented by $\mathcal{S}^{t}=\left[s_{1}, s_{2}, \ldots, s_{n}\right]$ stores the best experience of the individuals in each generation of the population space. Situational knowledge component is updated by $U$ pdate() function as follows:

$$
\mathcal{S}^{t+1}= \begin{cases}\mathbf{X}_{l}^{t} & \text { if } \quad f\left(\mathbf{X}_{l}^{t}\right)<f\left(\mathcal{S}^{t}\right), \quad l=1,2, \ldots, n_{\text {accept }} \\ \mathcal{S}^{t} & \text { otherwise }\end{cases}
$$

where $\mathbf{X}_{l}^{t}$ is the $l$ th accepted individual at iteration $t$ and $n_{\text {accept }}$ represents the number of accepted elite individuals to update the belief space.

\subsubsection{Normative knowledge}

The normative knowledge source, introduced by Chung, Reynolds (1998), indicates the standards to behaviour of the individuals in each dimension of the problem. This includes a set of intervals representing high quality or socially acceptable individuals, which specify appropriate search ranges in each dimension of the search space. These intervals or norms provide the guidelines for individuals in the population space to follow. Following the cultural norms plays an important role in human social intelligence (Clayton et al., 2000).

In CAs, the normative knowledge stores a set of information for each dimension of the problem, which can be mathematically expressed as follows: 


$$
\mathcal{N}^{t}=\left[\begin{array}{cccc}
I_{1}^{t} & I_{2}^{t} & \ldots & I_{n}^{t} \\
L_{1}^{t} & L_{2}^{t} & \ldots & L_{n}^{t} \\
U_{1}^{t} & U_{2}^{t} & \ldots & U_{n}^{t}
\end{array}\right]
$$

where $\mathcal{N}^{t}$ represents the normative knowledge source at iteration $t, I_{j}^{t}=$ $\left[x_{m i n, j}^{t}, x_{m a x, j}^{t}\right]$ indicates the belief interval of the $j$ th dimension of the problem, $x_{\min , j}^{t}$ and $x_{\text {max }, j}^{t}$ represent the lower and upper normative bounds of the $j$ th dimension of the problem, respectively, $L_{j}^{t}$ and $U_{j}^{t}$ are the fitness function values for the lower and upper normative bounds, respectively.

To update the normative knowledge source, CA uses an conservative approach to prevent excessive narrow belief intervals. The components of the normative knowledge source are updated by $U p d a t e()$ function in each generation as follows:

$$
\begin{aligned}
& x_{\min , j}^{t+1}= \begin{cases}x_{l, j}^{t} & \text { if } x_{l, j}^{t} \leq x_{\min , j}^{t} \quad \text { or } \quad f\left(\mathbf{X}_{l}^{t}\right)<L_{j}^{t} \\
x_{m i n, j}^{t} & \text { otherwise }\end{cases} \\
& x_{\text {max }, j}^{t+1}= \begin{cases}x_{l, j}^{t} & \text { if } x_{l, j}^{t} \geq x_{\text {max }, j}^{t} \quad \text { or } \quad f\left(\mathbf{X}_{l}^{t}\right)<U_{j}^{t} \\
x_{\text {max }, j}^{t} & \text { otherwise }\end{cases} \\
& L_{j}^{t+1}= \begin{cases}f\left(\mathbf{X}_{l}^{t}\right) & \text { if } \quad x_{l, j}^{t} \leq x_{m i n, j}^{t} \quad \text { or } \quad f\left(\mathbf{X}_{l}^{t}\right)<L_{j}^{t} \\
L_{j}^{t} & \text { otherwise }\end{cases}
\end{aligned}
$$

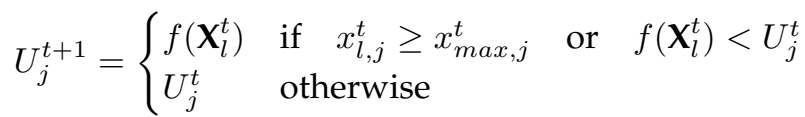

In the above equations, $l=1,2, \ldots, n_{\text {accept }}$ and $\mathbf{X}_{l}^{t}$ represents the $l$ th accepted individual at iteration $t$ and $x_{l, j}^{t}$ indicates its $j$ th variable.

\subsubsection{Topographical knowledge}

The topographical knowledge was introduced by Jin, Reynolds (1999a,b) as region-based schemata within the CAs, which is an multi-dimensional grid illustration of the solution space. The topographical knowledge source assumes the search space as a multi-dimensional grid or array composed of a set of cells in mesh expressed by $\mathcal{C}^{t}=\left[\mathbf{c}_{1}, \mathbf{c}_{2}, \ldots, \mathbf{c}_{k}\right]$, in which each element $\mathbf{c}_{i}$ represents the corresponding records for each cell and $k$ is the number of the belief cells in the topographical knowledge source. Each cell $\mathbf{c}_{i}$ in topographical knowledge stores a set of information, such as information about the feasibility and frequency of cell occupation. For example, Jin, Reynolds (1999a) represented each cell of the topographical knowledge source as follows:

$$
\mathbf{c}_{i}=\left[\operatorname{Class}_{i}, C n t 1_{i}, C n t 2_{i}, W_{i}, \operatorname{Pos}_{i}, \mathbf{C s i z e}_{i}\right]
$$

In Equation (8), Class $_{i}$ represents the information about the feasibility of cell $i$, including feasible,infeasible, semi-feasible or unknown; $C n t 1_{i}$ and $C n t 2_{i}$ indicate the number of feasible and infeasible individuals in cell $i$, respectively; $W_{i}$ is the weight of 
cell $i$, which indicates the quality of the cell; $\mathbf{P o s}_{i}$ represents the position of cell in the search space; Csize $_{i}$ denotes the size of the cell for all dimensions of the problem. It is worth mentioning that the type of the information stored in each cell of the topographical knowledge source depends on type of the optimisation problem. Broadly speaking, this knowledge source can be very useful to extract useful information about the search space and guide the search process. One of the crucial points in using topographical knowledge source during the solution finding process is the computational efficiency. Updating the cells of the topographical knowledge source can be computationally expensive, particularly when the cell sizes are small and the number of cells is huge. To remedy this inefficiency, $k-d$ ternary tree-based representations were used to describe the cells within the topographical knowledge source (Ali et al., 2016b; Peng, Reynolds, 2004; Ali et al., 2015).

\subsubsection{Domain knowledge}

The domain knowledge source $\mathcal{D}^{t}$ was first introduced by Reynolds, Saleem (2005) into CAs and is similar to the situational knowledge source. The domain knowledge source stores the positions of the best solutions obtained through different generations and forms an archive of the best individuals since the evolution started (Engelbrecht, 2007).

\subsubsection{History knowledge}

The history (or temporal) knowledge source $\mathcal{H}^{t}$ was proposed by Saleem to deal with the problems in which the search landscapes change during the evolution. This knowledge source maintains the shifts happened within the search regions during the solution finding process. The history knowledge stores the current best solution (i.e, the best individual before the last shift in the search regions), the directional change in the search region, and the distance change for each dimension of the problem. The data structure for the history knowledge is shown in Fig. 2. In Fig. 2, $\mathbf{e}_{i}$ represents the best current solution obtained before the last shift in the search regions, the list $\left(A_{d i r, 1}, A_{d i r, 2}, \ldots, A_{d i r, n}\right)$ indicates the directional change in the search region, and $\left.A_{\text {dire }, 2}, \ldots, A_{d i r, n}\right)$ represents the distance change, and $n$ is the number of variables of the problem.

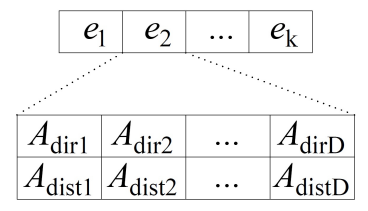

Figure 2: History knowledge structure (Ali et al., 2016b).

\subsection{Communication Protocol}

As mentioned earlier, the connection between the population and belief spaces is provided through communication protocols. CAs employ two functions as the communication protocols, including acceptance, update, and influence functions. The acceptance function determines which individuals in the population can affect the belief space, while the effect of the knowledge sources on the population space is determined by the influence function. These two functions are discussed in following subsections. 


\subsubsection{Acceptance function}

In order to update the knowledge sources of the belief space, a set of individuals demonstrating overall behaviour of the population space should be selected. Generally speaking, the acceptance functions used in literature can be classified into static and dynamic categories (Engelbrecht, 2007). In static acceptance functions, the number of accepted individuals is constant during the evolution process. It is very common to select a given percentage of the individuals, ranging from $1 \%$ to $100 \%$, to update the belief space depending on the performance of the algorithm in a given application (Chung, Reynolds, 1998). In the dynamic acceptance functions, the number of individuals selected to update the beliefs is not constant and varies from generation to generation. For example, Reynolds, Chung (1997a) proposed a fuzzy acceptance function to select the individuals to shape the beliefs. As another example, Ali et al. (2016b) used an dynamic acceptance function with reset mechanism, in which the number of accepted individuals decreases as generation counter increases.

\subsubsection{Influence function}

In a real human society, the individuals follow the global beliefs, which are acquired by previous generations and translated to the current generation. In order to model this phenomenon, CAs use the influence functions to affect the population space and create a new generation of individuals. The types of the influence functions depends on the problem type and utilised knowledge sources. When multiple types of knowledge sources are used, the main challenge in designing influence functions is to determine the extent of the impact of every single one of them on the population space. Reynolds, Chung (1997b) proposed four influence functions based on the situational and normative knowledge sources as follows:

- The first influence function uses only the normative knowledge source to determine the step size during the search process:

$$
x_{i, j}^{t+1}=x_{i, j}^{t}+\operatorname{size}\left(I_{j}^{t}\right) N_{i, j}(0,1) \quad j=1,2, \ldots, n
$$

where $x_{i, j}^{t+1}$ indicates the $j$ th element of the $i$ th individual at iteration $t+1$, $\operatorname{size}\left(I_{j}^{t}\right)=x_{\max , j}^{t}-x_{\min , j}^{t}$ represents the size of the normative interval for the $j$ th variable, and $N_{i, j}(0,1)$ is the random number generated by normal distribution with mean value of 0 and standard deviation of 1 .

- The second influence function determines the search direction based on the situational knowledge source:

$$
x_{i, j}^{t+1}=\left\{\begin{array}{lll}
x_{i, j}^{t}+\left|\sigma_{i, j}^{t} N_{i, j}(0,1)\right| & \text { if } & x_{i, j}^{t}<s_{j}^{t} \\
x_{i, j}^{t}-\left|\sigma_{i, j}^{t} N_{i, j}(0,1)\right| & \text { if } & x_{i, j}^{t}>s_{j}^{t} \\
x_{i, j}^{t}+\sigma_{i, j}^{t} N_{i, j}(0,1) & \text { Otherwise }
\end{array}\right.
$$

where $s_{j}^{t}$ is the $j$ th element of the situational knowledge at iteration $t$, and $\sigma_{i j}^{t}$ represents the strategy parameter of the $j$ th variable of $i$ th individual.

- The third influence function uses the situational knowledge to determine the search direction, while the step size is determined by based on the normative knowl- 
edge component. This influence function is similar to Equation 10, which can be stated as follows:

$$
x_{i, j}^{t+1}= \begin{cases}x_{i, j}^{t}+\left|\operatorname{size}\left(I_{j}^{t}\right) N_{i, j}(0,1)\right| & \text { if } x_{i, j}^{t}<s_{j}^{t} \\ x_{i, j}^{t}-\left|\operatorname{size}\left(I_{j}^{t}\right) N_{i, j}(0,1)\right| & \text { if } x_{i, j}^{t}>s_{j}^{t} \\ x_{i, j}^{t}+\operatorname{size}\left(I_{j}^{t}\right) N_{i, j}(0,1) & \text { Otherwise }\end{cases}
$$

- The fourth influence function determines both of the search direction and step size based on the normative knowledge source as follows:

$$
x_{i, j}^{t+1}= \begin{cases}x_{i, j}^{t}+\left|\operatorname{size}\left(I_{j}^{t}\right) N_{i, j}(0,1)\right| & \text { if } \quad x_{i, j}^{t}<x_{\min , j}^{t} \\ x_{i, j}^{t}-\left|\operatorname{size}\left(I_{j}^{t}\right) N_{i, j}(0,1)\right| & \text { if } x_{i, j}^{t}>x_{\max , j}^{t} \\ x_{i, j}^{t}+\beta \operatorname{size}\left(I_{j}^{t}\right) N_{i, j}(0,1) & \text { Otherwise }\end{cases}
$$

where $\beta>0$ is a constant parameter.

\subsection{Algorithmic steps of CAs}

Now, let us consider a minimisation problem of function $f\left(\mathbf{X}=\left[x_{1}, x_{2}, \ldots, x_{n}\right]\right)$, in which $n$ is the number of variables. The algorithmic steps of CAs for this problem can be summarised as follows:

Step 1: Initialisation of population space

In the first step, the population space $P S(t)$ of CA is initialised within the search space as follows:

$$
\mathbf{X}_{i, j}^{0}=\phi\left(l_{j}, u_{j}\right), \quad i=1,2, \ldots, N, \quad j=1,2, \ldots, n
$$

where $\mathbf{X}_{i, j}^{0}$ indicates the $j$ th element of $i$ th individual in the population space, $l_{j}$ and $u_{j}$ are the lower and upper bounds for the variable $j, \phi($.$) represents the uniform random$ function, and $N$ is the population size.

Step 2: Initialisation of belief space

In the second step, the iteration number $t$ is set to 0 and the initial belief space $\mathcal{B}^{0}=\left\{\mathcal{S}^{0}, \mathcal{N}^{0}, \mathcal{C}^{0}, \mathcal{D}^{0}, \mathcal{H}^{0}\right\}$ is initialised with appropriate values. For example, the normative knowledge source is initialised by assuming $x_{\min }^{0}=-\infty, x_{\max }^{0}=\infty, L_{j}^{0}=$ $\infty$, and $U_{j}^{0}=\infty$ as follows:

$$
\mathcal{N}^{0}=\left[\begin{array}{llll}
\infty & \infty & \ldots & \infty \\
\infty & \infty & \cdots & \infty \\
\infty & \infty & \cdots & \infty
\end{array}\right]
$$

Step 3: Fitness evaluation

In this step, the fitness functions of the individuals in the population space are evaluated.

Step 4: Update the belief space 
In the fourth step, the iteration number is set to $t=t+1$ and the knowledge sources of the belief space are updated by the information provided by the individuals of population space. For example, Equation (2) is used to update the situational knowledge source and Equations (4-7) are used to update the normative knowledge source.

Step 5: Influence the population space

Then, the information within the knowledge sources of the belief space are used to produce new generation of individuals $P S(t+1)$. For instance, the new generation of individuals can be determined based on the Equations (9-12).

Step 6: Stop or redo

Repeat steps 3-5 until the stopping criterion is reached and the optimal solution is yielded.

\section{Applications of CAs}

In recent decades, CAs have been applied to solve a wide variety of problems in science and engineering, such as civil engineering, mechanical engineering, chemical engineering, electrical engineering, and computer science. The numerical results in literature revealed the capabilities of CAs in solving different types of problems. Due to multi-disciplinary nature of the investigated problems in literature, it is quite tricky to categorise them into separate fields. However, this study tries to categorise them into mentioned fields. Table 2 lists the prominent applications of CAs in different areas. In the following subsections, a exhaustive summary of the applications of CAs to each of the mentioned areas will be provided.

Table 2: Applications of CAs and their variants in different fields.

\begin{tabular}{|c|c|}
\hline Application area & Authors \\
\hline \multicolumn{2}{|l|}{ Civil engineering } \\
\hline Transportation engineering & $\begin{array}{l}\text { Reyes et al. (2010), Liu et al. (2012), Arpaia et al. (2007), } \\
\text { Maldonado et al. (2013), Xue (2020) }\end{array}$ \\
\hline Structural engineering & $\begin{array}{l}\text { Jalili, Hosseinzadeh (2015), Jalili et al. (2019), Jafari et al. } \\
\text { (2019), Ali et al. (2018) }\end{array}$ \\
\hline Environmental engineering & Liu, Lin (2015) \\
\hline Urban engineering & Jayyousi, Reynolds (2014) \\
\hline Water engineering & Yan et al. (2017a), Gu, Wu (2010) \\
\hline \multicolumn{2}{|l|}{ Mechanical engineering } \\
\hline Damage detection & Guedria, Hassine (2018) \\
\hline Optimum engineering design & $\begin{array}{l}\text { Ali, Reynolds (2014),King et al. (2008), Santos Coelho dos, } \\
\text { Mariani (2006), Reynolds, Ali (2008b), Reynolds, Peng } \\
\text { (2005) }\end{array}$ \\
\hline Optimal vibration control & Wang et al. (2015) \\
\hline Vehicle safety & Kobti et al. (2006b) \\
\hline Flight control & Meng et al. (2011) \\
\hline Gas turbine operation & Pierezan et al. (2019) \\
\hline Renewable energy & Amalo et al. (2020) \\
\hline \multicolumn{2}{|l|}{ Chemical engineering } \\
\hline Process optimisation & Haikal, El-Hosseni (2011) \\
\hline Ammonia synthesis & Xu et al. (2010), Xu et al. (2012) \\
\hline
\end{tabular}


Table 2 - Continued from previous page

\begin{tabular}{l}
\hline Application area \\
\hline Molecular crystallography \\
Diesel blending \\
Electrical engineering \\
Design of high temperature su- \\
perconducting magnets \\
Economic dispatch
\end{tabular}

Network reconfiguration Sensor fault detection and correction

Wireless sensor networks Authors Tremayne et al. (2009) Gao et al. (2019)

Signal processing Maintenance scheduling Digital watermarking Power system stabilizer design Reactive power optimisation Optimal operation of cascade hydropower station Optimal design of passive power Filters

Hydrothermal scheduling Hydro production scheduling Multi-robot tasks allocation Robot soccer players Sea-surface weak targets detection

Image matching

Image detection

Fault detection

PID parameter optimisation

Frequency assignment

Gesture recognition

Chen et al. (2013)

Goudarzi et al. (2016), Zhang et al. (2013), Bhattacharya et al. (2012a), Santos Coelho dos et al. (2009), De Freitas et al. (2018), Ya-Li, Li-qing (2010), Goudarzi et al. (2017)

Haldar, Chakraborty (2014), Haldar, Chakraborty (2015) Khan et al. (2014), Khan et al. (2017)

Guo et al. (2013b), Guo et al. (2013a), Kulkarni, Desai (2020), Murugadass, Sivakumar (2020)

Gao, Diao (2010), Gao, Diao (2011), Gao, Xu (2013)

Digalakis, Margaritis (2002)

Gao et al. (2017a)

Khodabakhshian, Hemmati (2013), Tavakoli et al. (2014)

Bhattacharya et al. (2012b)

Wang et al. (2009), Xie et al. (2009)

Guo et al. (2008)

Yuan, Yuan (2006), Lu et al. (2011), Yuan et al. (2009)

Yuan et al. (2008)

Yu et al. (2011)

Reynolds, Chung (1997c), Ali et al. (2014b)

Cai et al. (2018)

Yan et al. (2017b)

Wang et al. (2017)

Pan et al. (2010)

Xiao et al. (2010)

Alami, El Imrani (2008)

Waris, Reynolds (2015)

\section{Computer science}

Function optimisation

Chung, Reynolds (1998), Kobti, others (2014), Kobti, others (2013), Becerra, Coello (2004), Coello, Becerra (2002b), Xue et al. (2011), Guo et al. (2011b), Gao et al. (2006a), Nguyen, Yao (2008), Gao et al. (2006b), Gao et al. (2007), Nguyen, Yao (2006), Li et al. (2010), Ali et al. (2011a), Guo et al. (2018), Guo et al. (2011c), Reynolds, Zhu (2001), $\mathrm{He}, \mathrm{Xu}$ (2011), Sun et al. (2010), Sun et al. (2012), Dixit et al. (2019), Awad et al. (2017), Ali et al. (2016a), Ali, Awad (2014), Ali et al. (2012), Xue, Guo (2007), Coello, Becerra (2002a), Ali et al. (2016b), Alami et al. (2007), Huang et al. (2008), Wu et al. (2010), Stanley et al. (2019), Tang, Li (2008), Awad et al. (2013), Coello, Becerra (2003), Zhou et al. (2019), Reynolds, Kinnaird-Heether (2017a), Reynolds, Kinnaird-Heether (2017b), Ali et al. (2014a), Reynolds, Gawasmeh (2012), Ali et al. (2011b), Ali, Reynolds (2009),Reynolds, Ali (2008c),Chung, Reynolds (1996b), Mao et al. (2020), Murugadass, Sivakumar (2020), Singh et al. (2018),Muhamediyeva (2020)

Continued on next page 
Table 2 - Continued from previous page

\begin{tabular}{|c|c|}
\hline Application area & Authors \\
\hline Job shop problem & $\begin{array}{l}\text { Cortés Rivera et al. (2007), Zhang, Zhu (2012), Kobti, oth- } \\
\text { ers (2012), Yang, Gu (2014), Becerra, Coello (2005), Niu } \\
\text { et al. (2011), Ho, Tay (2004) }\end{array}$ \\
\hline Facility location & Lagos et al. (2014), Cabrera et al. (2011) \\
\hline Classification & $\begin{array}{l}\text { Srinivasan, Ramakrishnan (2012), Yan et al. (2016), Srini- } \\
\text { vasan, Ramakrishnan (2013b), Srinivasan, Ramakrishnan } \\
\text { (2013a), Srinivasan, Muruganandam (2020) }\end{array}$ \\
\hline Set covering & Crawford et al. (2013), Crawford et al. (2007) \\
\hline Expert systems & Sternberg, Reynolds (1997) \\
\hline Semantic network & $\begin{array}{l}\text { Rychtyckyj, Reynolds (2005), Rychtyckyj, Reynolds } \\
\text { (2000), Rychtyckyi, Reynolds (1999),Rychtyckyj, Reynolds } \\
\text { (2002),Rychtyckyj, Reynolds (2001),Rychtyckyj, Reynolds } \\
\text { (1998) }\end{array}$ \\
\hline Program understanding & Reynolds, Sverdlik (1995) \\
\hline Timetabling & Soza et al. (2011), Soza et al. (2007) \\
\hline Mobile agent routing & Jun et al. (2008) \\
\hline Ancient landscape exploration & Vitale et al. (2011b) \\
\hline Web search & Zhang (2012) \\
\hline Social networks & $\begin{array}{l}\text { Zadeh, Kobti (2015), Selvarajaha et al. (2019), Selvarajah } \\
\text { et al. (2020) }\end{array}$ \\
\hline Fake news detection & Shah, Kobti (2020) \\
\hline Intelligent logistics & Ochoa et al. (2010) \\
\hline Site location decision-making & Reynolds, Nazzal (1997) \\
\hline Symbolic regression & Zannoni, Reynolds (1997) \\
\hline Neuro-fuzzy systems & Chen, Yang (2015), Chen et al. (2008) \\
\hline $\begin{array}{l}\text { Multi-aircraft cooperative target } \\
\text { allocation }\end{array}$ & Li et al. (2012) \\
\hline Travelling salesman problem & $\begin{array}{l}\text { Unold, Tarnawski (2016), Unold, Tarnawski (2017), Kim, } \\
\text { Cho (2009) }\end{array}$ \\
\hline $\begin{array}{l}\text { Partially observable Markov de- } \\
\text { cision process }\end{array}$ & Prestwich et al. (2008) \\
\hline Support vector regression & Cheng et al. (2009) \\
\hline Data mining & $\begin{array}{l}\text { Abdolrazzagh-Nezhad (2020), Jin, Reynolds (2000), } \\
\text { Reynolds, Al-Shehri (1998),Reynolds, Al-Shehri (1997), } \\
\text { Deng et al. (2020) }\end{array}$ \\
\hline Cloud computing & Mojab et al. (2019) \\
\hline Fog computing & Hosseinioun et al. (2020) \\
\hline Neural fuzzy network design & Lin et al. (2009) \\
\hline Feature selection & Oloruntoba et al. (2019) \\
\hline Dairy product demand & Goli et al. (2019) \\
\hline Online marketing & Selvarajah et al. (2019) \\
\hline Quality of service & Sun et al. (2009) \\
\hline Virtual machine placement & Mohammadhosseini et al. (2019) \\
\hline Evolutionary programming & $\begin{array}{l}\text { Coello Coello, Becerra (2004), Ostrowski, Reynolds (2003), } \\
\text { Cowan, Reynolds (1999), Chung, Reynolds (1996b) }\end{array}$ \\
\hline Games & $\begin{array}{l}\text { Vitale et al. (2011a), Fogarty et al. (2011), Reynolds, } \\
\text { Kinnaird-Heether (2003) }\end{array}$ \\
\hline Software testing & Ostrowski, Reynolds (1999) \\
\hline Image segmentation & Reynolds, Rolnick (1995); Reynolds, Rolnick (1995) \\
\hline Boolean circuits & Reynolds, Sverdlik (1993) \\
\hline City security & Ochoa et al. (2012) \\
\hline \multicolumn{2}{|l|}{ Others } \\
\hline Anthropology & Kobti et al. (2006a) \\
\hline Economic recession & Ostrowski, Reynolds (2005) \\
\hline Virtual enterprise & Wei, Yan-Ping (2012),Ostrowski, Reynolds (2004) \\
\hline
\end{tabular}


Table 2 - Continued from previous page

\begin{tabular}{ll}
\hline Application area & Authors \\
\hline Archaeology & Reynolds et al. (2008a) \\
Biological science & Alexiou, Vlamos (2012), Judeh et al. (2014), Judeh et al. \\
& (2013) \\
Industrial engineering & Terán et al. (2017), Rychtyckyj et al. (2003) \\
Physics & Santos Coelho dos, Alotto (2009), Pan et al. (2016) \\
\hline
\end{tabular}

\subsection{Civil engineering}

CAs have found some applications in civil engineering areas. The application studies of CAs in civil engineering area can be categorised into five sub-disciplines as follows: i) transportation engineering, ii) structural engineering, iii) environmental engineering, iv) urban engineering, and v) water engineering.

In the transportation area, Reyes et al. (2010) employed CA to find the optimal route between two points in the urban public transportation of the Leon city located in Mexico, in which the population has been significantly increased and caused the inhabitants to waste their time on travelling with public transportation. Authors investigated a given part of the city with a set of buses and stations as the case study. Simulation results indicated that $\mathrm{CA}$ is able to generate better transportation paths than handbook estimates done by experts. In another study, Liu et al. (2012) applied CA to find shortest path between two points in vehicle routing problem, in which the weather, road quality, traffic, and other uncertain factors were considered. The numerical results obtained from an urban road traffic network showed the capability of CA in finding shortest path. Arpaia et al. (2007) employed CA with DE operators for industrial fault isolation. Experiments were done on industrial problems, such as motor-vehicle fleet remote monitoring and automatic fault isolation of vehicle wear, operating danger, and fraud in a company that transports dangerous goods. Maldonado et al. (2013) developed an android software based on CA and Data Mining to determine the probability of occurring a traffic accident in a selected part of the city. This software can prevent the unnecessary congestion within the cities and reduce the number of traffic accidents in the long term. Xue (2020) employed a CA-based CS algorithm for time-dependent vehicle routing problem with stochastic customers, in which the traffic speed is estimated by an adaptive fractional Kalman filter. Authors investigated a real case study with different business districts in Xiamen, China. Although the literature review revealed the above-mentioned practical applications of CAs in transportation engineering, the performance of them have not yet tested on other types of optimisation problems in traffic engineering, such as signal optimisation (Gao et al., 2017b; Nallaperuma et al., 2020; Liang et al., 2020), battery charging optimisation of electrical vehicles (Karakatič, 2020), urban network design (Gallo et al., 2010), train scheduling (Samà et al., 2015), and transport energy modeling (Ceylan et al., 2008).

In the field of structural engineering, Jalili, Hosseinzadeh (2015) applied CA for optimal design of truss structures under stress and displacement constraints. Authors verified the efficiency of $C A$ through optimising four real-world truss structures. Comparison results between CA and other meta-heuristics, such as BB-BC, PSO, and GA, illustrated that $\mathrm{CA}$ is able to provide relatively better structural designs. In another research, Jalili et al. (2019) introduced an improved CA (ICA) for simultaneous size and 
shape optimisation of dome-shaped structures under static and frequency constraints. Structural optimisation under frequency constraints is a highly non-linear optimisation problem. Authors investigated the size and layout optimisation of a 276-bar lamella dome structure under stress and displacement constraints and a 708-bar lamella dome structure under frequency constraints. Jafari et al. (2019) developed a hybrid CA and $\mathrm{EHO}$ for optimum design of truss structures. Authors applied their algorithm to solve some benchmark structural design problems, including 10-bar truss, 25-bar truss, 72bar truss, and 120-bar dome structures. The results indicated that the standard CA was able to provide better structural designs than $\mathrm{EHO}$ algorithm for all design examples. Ali et al. (2018) presented a balanced fuzzy CA with a modified Levy flight search to solve real-world parameter optimisation. Authors tested the performance of the approach on a set of benchmark functions and a 120-bar dome structure.

In the field of the environmental engineering, Liu, Lin (2015) proposed a CA-based approach to solve the spatial forest resource planning problem, in which the maximising total timber volume harvested over a harvest planning schedule was considered as the objective function. Authors performed a statistical comparison between the CA and SA algorithm by assuming different values for maximum iterations and population sizes. Their statistical comparisons revealed that CA is generally performs better than SA algorithm in terms of efficiency and stability of the results.

CA was also applied to the urban engineering. Jayyousi, Reynolds (2014) applied CA to produce high level structural functional models of cities, in which each individual of CA competes to build up a high level model of the site from a set of building blocks. Their study proved that the application of CA to urban planning can provide new insights into the plans produced by human experts.

In the field of the water engineering, $\mathrm{Gu}, \mathrm{Wu}(2010)$ used CA to solve multiobjective water resources optimisation problem, in which the correlation of power generation between reservoirs and downstream areas was considered. In another study, Yan et al. (2017a) employed CA to solve the contaminant source identification problem in water distribution networks by assuming different sizes of water supply networks. Authors evaluated the performance of CA based on three water supply networks with 129,430 , and 12,527 nodes. Optimisation results revealed that CA is able to find the approximate pollutant source location with an acceptable level of accuracy. However, it seems that its performance needs to be improved to identify the pollutant sources more accurately in larger scales of water distribution networks.

\subsection{Mechanical engineering}

CA has also been employed to find solutions for the optimisation problems in mechanical engineering. Guedria, Hassine (2018) applied CA to solve the target optimisation problem of vibration-based damage detection. The performance of CA was investigated on the single and multiple damage detection of a cantilevered beam. Comparisons between CA and other algorithms, such as PSO and DE, showed the superiority of $\mathrm{CA}$ in accurately detecting the exact locations and the extents of damages under measurement noise. As the cantilever beam is a relatively simple structure, the performance of CA needs to be investigated on the damage detection of more complicated large-scale structures (Ghiasi et al., 2019; Perera, Ruiz, 2008).

In several studies, CAs have been utilised to solve the mechanical engineering 
design problems. Ali, Reynolds (2014) introduced a hybrid CA and TS (CA-TS) algorithm to solve the complex non-linear constrained engineering design optimisation problems. The results reported by authors show that the standard CA performs slightly better than TS. Similarly, King et al. (2008), Reynolds, Ali (2008b), and Reynolds, Peng (2005) applied CA toolkit to solve mechanical engineering design problems.

The vibration control, vehicle safety, and flight control are other interesting applications of CAs in mechanical engineering. Wang et al. (2015) introduced an optimal vibration control strategy for vehicle's active suspension by combining CA and niche algorithm to design Fuzzy-PID controller and optimise control rules. Simulation results revealed that the optimised control rules can improve the ride comfort and handling stability of automobile. However, the convergence curve of CA reported for this problem shows that the CA converged to the solution within 23 generations, which is likely a sign of premature convergence. Kobti et al. (2006b) applied CA to guide driver learning to improve its performance in terms of the child vehicle safety and the injury level. Authors have considered a social networks between the drivers, in which the drivers can exchange their knowledge between each other. Meng et al. (2011) used a CA based on the PSO (CAPSO) to flight control law clearance.

Another interesting application area of meta-heuristics in mechanical engineering is in the performance enhancement of industrial gas turbines (or heavy-duty turbines). Pierezan et al. (2019) employed Cultural COA (CCOA) to improve the performance of heavy-duty gas turbines of a combined cycle power-plant located in Brazil, in which the gas consumption is minimised by taking into account the pollutant emission regulations and the turbine's physical limitations.

\subsection{Chemical engineering}

CAs have found some interesting applications in chemical engineering area. Haikal, ElHosseni (2011) developed a modified cultural-based GA (MCBGA) to solve two chemical engineering optimisation problems. Authors solved the optimal control problem of a fed-batch fermentor for Penicillin production, in which maximisation of Penicillin production is considered as the objective function. Haikal, El-Hosseni (2011) also investigated the effectiveness of their algorithm in optimising the performance of the Isothermal continuous stirred tank reactor, which is a common model for a chemical reactor in chemical engineering. The statistical comparisons provided by the authors show that the MCBGA approach performed better than rival methods in terms of best, average, worst, and standard deviation of the results.

$\mathrm{Xu}$ et al. (2010) and $\mathrm{Xu}$ et al. (2012) proposed a multi-population cultural DE (MCDE) algorithm for optimising the chemical process of Ammonia synthesis. Ammonia has important applications in producing fertilizers in agricultural sector. Authors formulated the Ammonia Synthesis as a optimisation problem, in which the maximising net value of Ammonia is treated as the objective function. Tremayne et al. (2009) applied a hybrid cultural DE (CDE) algorithm to solve the problem of crystal structure determination from powder diffraction data. The main goal of the crystal structure determination is to find the precise spatial arrangements of all of the atoms in a chemical compound in the crystalline state (Massa, 2013). In Ref. (Tremayne et al., 2009), authors performed a kind of sensitivity analysis of internal parameters of the algorithm in order to find the best combination of them. Gao et al. (2019) used a hybrid cultural HS algorithm to optimise diesel blending procedure, in which a series of operations and 
units are performed to transform crude oils into various products.

\subsection{Electrical engineering}

One of the interesting engineering application areas of CAs is electrical engineering. Chen et al. (2013) formulated the design of the high temperature superconducting magnets as the optimisation problem and suggested CA to solve it. In comparison to the conventional design method, the simulation results showed that the approach is able to provide high temperature superconducting magnets with better volume of solenoid coils. According to the results, CA is computationally efficient than the conventional GA for this problem, as it requires less computational time.

EDP is one of the attractive application areas of CA in electrical engineering. The main objective of this problem is minimising total fuel cost of a number of electricity generation facilities to allocate system load demand under transmission and operational constraints. Bhattacharya et al. (2012a) combined CA with EP to solve EDPs. In order to find the feasible solutions, authors used a map of feasible regions of search space to guide the search process. In comparison to PSO, GA, EP, and DE algorithms, the results yielded from a 13-generator system with valve point effects indicated that the CA is able to provide lower optimal fuel costs. Santos Coelho dos et al. (2009) proposed an improved DE approach based on CA and diversity measure to solve EDPs. Authors demonstrated the efficiency of their algorithm on two test systems consisting of 13 and 40 thermal generators. Ya-Li, Li-qing (2010) applied improved cultural PSO algorithm to solve EDP of 3-generators unit with 6 plants under transmission losses and valve-point loading.

In order to consider the released environmental pollutants by the systems of electricity generation through the thermal power plants, researchers have been added the emission effects to the EDP. Goudarzi et al. (2016) suggested four versions of CAs with different influence functions to solve the combined environmental EDP, in which minimising both of the fuel cost and emissions are considered as the objective functions, simultaneously. By using a price penalty factor, authors merged these two objective functions into a single objective function and considered various power system constraints, such as the ramp-rate limit, emission costs, valve-point effect, prohibited operation zone, and the transmission losses. Comparing to other methods in literature, simulation results obtained from three different test case systems demonstrated that CAs are capable to solve combined environmental EDP more efficiently. The convergence diagrams provided by the authors illustrate that two versions of $\mathrm{CA}$ are able to converge the optimal solution faster than FA, BBO, ABC, PSO, and GA methods. Similar results were also reported in Ref. (Goudarzi et al., 2017) for this problem. Zhang et al. (2013) developed an enhanced multi-objective CA approach to solve environmental EDP under various constraints, including valve-point effect, prohibited operation zones, and transmission losses. The performance of CA was verified through a sixunit and ten-unit test systems. The comparisons between the non-dominated solutions obtained by the CA, NSGA-II and MODE algorithms indicated that not only CA provides better distributions of Pareto front for environmental EDP, but also requires fewer amount of computational time. De Freitas et al. (2018) suggested combined CA methods with local search techniques for environmental EDPs, in which the fuel cost and gas emission are considered as the objective functions. The performances of the algorithms were investigated by using a real power plant with 10 generators and a system 
with 13 generating units. The convergence histories illustrated that the standard CA converged to better total cost values than the standard GA.

Reducing the real power loss of the power system networks through network reconfiguration and capacitor installation is another interesting research area in electrical engineering. Haldar, Chakraborty $(2014,2015)$ introduced a modified CA (MCA) to solve the network reconfiguration and capacitor allocation problems for minimum real power loss. The performance of MCA was investigated on the standard IEEE 16 bus distribution network and a practical transmission network. According to the experiments, MCA was able to provide lower costs for the transmission network and distribution system in comparison to PSO and rcGA methods.

Some applications of CA can be found in the field of the sensor fault detection and correction. Khan et al. (2014) suggested a symmetric sensor failure technique with a hybrid CA and DE (CADE) algorithm for the correction of faulty arrays. In another research, Khan et al. (2017) applied CADE to detect fully and partially defective sensors in a linear array. Authors proposed a symmetrical structure of a linear array to reduce the computational complexity and the partial faults are detected by CADE approach. Numerical results obtained by these studies showed the efficiency of CADE algorithm. Guo et al. (2013b) proposed a quantum-inspired CA method for the coverage optimisation of wireless sensor networks. Simulation results verify the efficiency of the approach in comparison to other algorithms. In another study, Guo et al. (2013a) extended the quantum-inspired CA for the multi-objective energy-efficient coverage optimisation of wireless sensor networks, in which the network coverage rate and the node redundancy rate are considered as the objective functions. The results demonstrated that the approach is able to yield a better pareto-optimal solutions than other multiobjective approaches. Kulkarni, Desai (2020) applied CA for anchor-assisted, rangebased, multi-stage localisation of sensor nodes of wireless sensor networks. The results comparisons between $\mathrm{CA}, \mathrm{ABC}$, and PSO revealed the superiority of CA in terms of accuracy and computing time.

CA has been also applied to the signal processing. Gao, Diao (2010) used a differential cultural (DC) algorithm to design finite impulse response and infinite impulse response digital filters. Gao, Diao (2011) suggested a cultural firework (CF) algorithm for digital filters design, in which the firework algorithm and CA are combined. Authors investigated the problem of designing finite impulse response and infinite impulse response digital filters via CF algorithm. Gao, Xu (2013) proposed cultural quantuminspired SFLA for direction finding of non-circular signals. The results showed that the approach can be efficiently used to direction finding of coherent and non-coherent sources of non-circular signals.

Digalakis, Margaritis (2002) presented a parallel co-operating CA (PARCA) method to solve the electrical generator maintenance scheduling problem, in which sum of the overall fuel cost and the overall cost of maintenance is considered as the objective function. By combining CA and IWO algorithm, Gao et al. (2017a) developed a cultural invasive weed algorithm (CIWA) for optimising the robustness and the imperceptibility of the watermarking system, in which the copyright of image data is protected.

Khodabakhshian, Hemmati (2013) applied CAs for tuning parameters of power system stabiliser to improve overall system stability. Simulation results of two typical multi-machine electric power systems containing system parametric uncertainties and 
various loading conditions verify the efficiency of CAs in improving dynamic stability of the systems. The convergence diagrams and computing times reported by authors for CA and GA methods shows the superiority of CA. Tavakoli et al. (2014) employed $\mathrm{CA}$ to design the controllers of the shunt flexible AC transmission system and power system stabiliser coordinately. The numerical results and convergence comparisons between CA, GA, and BFA showed that CA was able to find better solutions within early stages of evolution process. Bhattacharya et al. (2012b) introduced a CA with a single point crossover to solve the reactive power optimisation problem. Wang et al. (2009) applied a chaos cultural PSO (CCPSO) algorithm for the optimal operation of cascade hydropower station. Xie et al. (2009) used a PSO based on CA to solve short-term optimal operation problem of cascade hydropower stations. Authors used the penalty function method to handle the constraints of the problem. Guo et al. (2008) used multiobjective CAs for optimal design of passive power filters by assuming the total harmonics distortion and cost for equipment as the objective functions. The simulation results indicated that the filter designed by CAs has better harmonics suppression effect and lower investment for equipment than the traditional experience-based filter design.

Some researches have been investigated the performance of CA in optimal scheduling of hydrothermal power systems. For example, Yuan, Yuan (2006) utilised $\mathrm{CA}$ to solve the daily generation scheduling of hydrothermal power systems. Comparison results between CA, Lagrange, and GA methods verified the fast convergence properties of CA in solving the optimal daily generation scheduling problem of hydrothermal systems. However, the comparison between CA and GA was not supported by a fair statistical analysis. In another work, Lu et al. (2011) proposed a hybrid multiobjective CA for short-term environmental/economic hydrothermal scheduling problem, in which CA and DE are combined. The efficiency of the method was investigated on four coupled hydro plants and three equivalent thermal plants with non-smooth valve-point effects cost function. Yuan et al. (2008) applied CA to solve hydro production scheduling problem in electricity market, in which the profit of the hydro company from selling energy is maximised. In a similar study, Yuan et al. (2009) employed CA to deal with the short-term generation scheduling of hydrothermal systems.

CAs have been applied to solve different problems in the field of the robotics. Yu et al. (2011) employed a heterogeneous interactive cultural hybrid algorithm (HICHA) to solve multi-robot exploration mission planning problem. In their approach, the spatial orthogonal allocation algorithm was used for getting max-min task allocation. Then, the best route was obtained by HICHA. Reynolds, Chung (1997c) applied CA to increase the individual skills and cooperative strategies among a team of autonomous robot soccer players, in which authors assumed a set of constraints on the behaviour of players. Ali et al. (2014b) used CA to evaluate the tactics of robotic soccer team, in which the coordination of groups of agents in complex multi-agent dynamic environments was optimised. Authors evaluated the performance of CA against GA and showed that CA-team performs better than GA-team.

Some other applications for CA in electrical engineering can also be found in literature. Cai et al. (2018) proposed a time-frequency distribution fusion strategy assisted by CA to improve the sea-surface weak targets detection performance of the marine surface surveillance radar systems. Authors reported the advantages of CA method in solving this problem. Yan et al. (2017b) introduced a hybrid isolation niche technology and CA to solve image matching problem. The efficiency of the approach 
was demonstrated by solving a image matching problem. According to the results reported by the authors, CA provided better match correct rates than those obtained by GA. Wang et al. (2017) applied an adaptive CA with improved quantum-behaved PSO (IQPSO) to detect underwater sonar images. Pan et al. (2010) adapted CA for the size minimisation of binary decision diagrams, in which a binary decision diagram is a directed acyclic graph. Binary decision diagrams are capable of providing canonical representation for Boolean functions and they have important applications in design and verification of digital systems. Authors evaluated the performance of CA against GA on a set of benchmark circuit fault detection problems. Numerical results verified the effectiveness of CA in finding smaller size of binary decision diagram. Xiao et al. (2010) employed a cultural based ant colony algorithm to parameter optimisation of PID controllers, which have important applications in industry process control. Alami, El Imrani (2008) applied a hybrid CA and EP method to solve the fixed spectrum frequency assignment problem, in which the objective is the minimising interference of an assignment plan to efficient use of radio spectrum.

\subsection{Computer science}

CAs have been attracted much attention to solve a wide range of problems in the field of the computer science, such as function optimisation, Job Shop Problem (JSP), facility location, classification, and set covering. In the following subsections, the references related to these problems will be discussed.

\subsubsection{Function optimisation}

One of the research areas where the application of CAs is most extensive is the function optimisation. Various researchers have been employed CAs and their variants to solve the unconstrained and constrained function optimisation problems (Chung, Reynolds, 1998; Kobti, others, 2014, 2013; Becerra, Coello, 2004; Coello, Becerra, 2002b; Xue et al., 2011; Guo et al., 2011b; Gao et al., 2006a; Nguyen, Yao, 2008; Gao et al., 2006b, 2007; Nguyen, Yao, 2006; Li et al., 2010; Guo et al., 2018; Ali et al., 2011a; Guo et al., 2011c; Reynolds, Zhu, 2001; He, Xu, 2011; Sun et al., 2010, 2012; Dixit et al., 2019; Awad et al., 2017; Ali et al., 2016a, 2012; Xue, Guo, 2007; Coello, Becerra, 2002a; Ali et al., 2016b; Alami et al., 2007; Huang et al., 2008; Wu et al., 2010; Stanley et al., 2019; Tang, Li, 2008; Awad et al., 2013; Coello, Becerra, 2003; Zhou et al., 2019; Reynolds, KinnairdHeether, 2017a,b; Ali et al., 2014a; Reynolds, Gawasmeh, 2012; Ali et al., 2011b; Ali, Reynolds, 2009; Reynolds, Ali, 2008c; Chung, Reynolds, 1996b; Mao et al., 2020; Murugadass, Sivakumar, 2020; Singh et al., 2018; Muhamediyeva, 2020).

\subsubsection{JSP}

Several researchers applied CAs for solving JSP, in which a set of jobs should be processed through a set of machines. The objective function is the minimisation of the total time required to perform the jobs under some constraints. Cortés Rivera et al. (2007) and Becerra, Coello (2005) employed CA as an alternative optimisation algorithm to solve JSP. Comparison results between CA and rival algorithms, such as GA and its hybridised version with local search, obtained from a set of benchmark JSPs revealed that $\mathrm{CA}$ is capable of generating competitive results for this problem. Their results showed that CA is able to provide better results than the standard GA, whereas its performance is comparable to GA with local search. Zhang, Zhu (2012) suggested a cultural PSO al- 
gorithm to minimise the maximum completion time in JSP. Kobti, others (2012) applied a multi-population version of CA to solve the JSP and indicated that the convergence speed of CA is remarkable. Yang, Gu (2014) developed a cultural-based genetic tabu algorithm for multi-objective JSP. Niu et al. (2011) employed a CA based on DE (CADE) algorithm to solve the hybrid flow shop scheduling problems with fuzzy processing time. Authors considered the makespan as the objective function, which is modeled as a triangular fuzzy number. The results reported for different instances showed that the standard CA performs statistically better than PSO method. Ho, Tay (2004) applied CA to solve the flexible JSP with re-circulation. Authors indicated the efficiency of CA in comparison to the conventional composite dispatching rule algorithm.

\subsubsection{Facility location}

The main aim of the facility location problem is to find the optimum placement of facilities of companies to reduce the transportation costs of product distribution between their costumers. Lagos et al. (2014) and Cabrera et al. (2011) applied CAs to solve a biobjective uncapacitated facility location problem, in which the cost minimisation of facility installation and customer allocation as well as maximisation of the customers coverage are considered as the objective functions. Comparing to NSGA-II, the results reported in these references for different benchmarks shows that the CA is capable of generating better solutions with less computational effort.

\subsubsection{Classification}

Briefly speaking, classification problems deal with the assigning data into one of several known classes. Srinivasan, Ramakrishnan (2012) employed CAs for multi-objective optimisation of classification rules. Yan et al. (2016) proposed a double weighted Naive Bayes and niching CA for multi-lable classification. Srinivasan, Ramakrishnan $(2013 \mathrm{~b}, \mathrm{a})$ proposed extended CA for multi-objective optimisation of classification rules. Their results showed that the extended CA can provide more accurate results than other methods, such as NSGA-II. Recently, Srinivasan, Muruganandam (2020) employed CA to solve multi-objective problem of rule pruning in classification algorithms, in which the unwanted rules are removed to reduce the difficulty of decision making process.

\subsubsection{Set covering}

The main aim of the set covering problem is to identify the smallest mutually independent subsets of a given set while minimising a cost function defined as the sum of the costs associated to each of the eligible subsets (Chu, Beasley, 1998). Crawford et al. $(2007,2013)$ applied CA to solve set covering problem. The costs obtained for different benchmarks revealed that CA performs remarkably better than GA and ACO methods.

\subsubsection{Others}

CAs have also interesting applications in some other types of problems in the field of the computer science. For instance, Reynolds, Sverdlik (1995) applied CA for program understanding, in which CA used to extract functional knowledge from a program object. Sternberg, Reynolds (1997) utilised CA for fraud detection in insurance companies, in which the fraudulent claims received by the insurance companies are 
identified to reduce the fraud losses. The results showed that CA has an efficient self-adaptive capability in dealing with dynamic optimisation problems. Rychtyckyi, Reynolds (1999) applied CA to solve the problem of semantic network reformulation by analysing the selection of significant attributes for subsumption. Rychtyckyj, Reynolds (1998) suggested a CA-based approach for semantic network reformulation. Rychtyckyj, Reynolds $(2000,2005)$ suggested CA to improve the performance of the subsumption algorithm in re-engineering large-scale semantic networks. Authors reported that $\mathrm{CA}$ is able to provide comparable results to those provided by the manual inspections. It seems that the superiority of CA over manual inspection is more obvious for more complex networks. Rychtyckyj, Reynolds $(2002,2001)$ applied CA to re-engineer the direct labour management system semantic network at Ford company from a top-down perspective. Authors indicated that CA is able to provide significantly better results comparing to the previously derived results through manual inspections.

Nowadays, the social media has an significant effect on our daily life, in which spreading the fake news generated by anonymous users can make a big challenge for the government and individuals in society. Recently, Shah, Kobti (2020) employed CA to identify the fake news using the text and images in social media. In comparison to the state-of-the-art methods, such as ANN-based methods, the results revealed that CA is able to detect the fake news with higher level of accuracy. Based on CA, Zhang (2012) presented a framework for evolutionary systems to provide an efficient search mechanism for finding useful contents on the web. Zadeh, Kobti (2015) proposed a knowledge-based evolutionary algorithm by using a multi-population CA to solve community detection in social networks. Reynolds, Nazzal (1997) used CAs to discover networks of sites based on the historical database.

Soza et al. $(2011,2007)$ applied CA to solve timetabling problems. Authors reported that CA performs weaker than SA for timetabling problems. Jun et al. (2008) employed an integrated CA and SA to solve the routing problem of the mobile agent. Vitale et al. (2011b) used CA for ancient landscapes exploration under the lake. Selvarajaha et al. (2019) applied CA to solve the problem of identifying a team of experts in social networks. Numerical results suggested that the efficiencies of CA and GA for this problem are almost same in terms of quality of the results and required computational effort. In a similar study, Selvarajah et al. (2020) used CAs to solve the team formation problems in the industrial organisational settings, in which the objective is forming group of teams with different skills in a social network to accomplish a set of projects. It seems that CA performed better than GA and other algorithms for higher number of projects. Ochoa et al. (2010) developed a software tool to provide an intelligent logistic service for purified water distribution among the costumers, in which the CA was used as optimiser. Authors combined data mining with CA to minimise the delivery costs.

Chen, Yang (2015) designed the neuro-fuzzy system by a DE-based symbiotic CA to solve the nonlinear control problems. Li et al. (2012) employed a hybrid CA and GA, called cultural-GA, to solve the multi-aircraft cooperative target allocation problem, in which the main aim is to select an optimal and reasonable weapon-target allocation plan to achieve the best combat effect.

Unold, Tarnawski $(2016,2017)$ proposed parallel cultural ACO and multipopulation CA to solve the travelling salesman problem. Kim, Cho (2009) applied a hybrid CA with local search to solve the travelling salesman problem. Ochoa et al. 
(2012) developed a mobile device based on CA and data mining, which is provided to analyse the risk of being in a part of city. The results showed that the integrated CA and data mining method can perform significantly better than the purely data mining approach.

Prestwich et al. (2008) applied CA to solve partially observable markov decision processes. From the reported diagrams in this reference, it seems that CA can converge to the optimal solutions faster than GA. Abdolrazzagh-Nezhad (2020) applied an enhanced CA to solve the multi-objective attribute reduction problem, which is an prepossessing problem in data mining. Authors provided a fair comparison of CCA against other meta-heuristic algorithms by investigating their performance with different population sizes. The reported resulted showed that CA provides better results than those provided by binary GA, PSO, ACO, ABC, and TS. Jin, Reynolds (2000) used CAs to mine knowledge in large-scale databases, in which the data mining is performed within the belief space and the optimisation is performed by the population space. The approach extracted some new patterns from a large-scale archeological database, which were unknown before. Reynolds, Al-Shehri $(1997,1998)$ tried to guide the incremental learning decision trees, which have important applications in representation of the results obtained from the data mining.

Oloruntoba et al. (2019) used a clan-based CA for feature selection, which is a process of selecting a subset of relevant features from data sets and has important applications in machine learning. Mojab et al. (2019) proposed an improved CA to solve the general big data workflow scheduling problem, in which the characteristics of cloud computing are optimally used to transfer large amounts of data. Goli et al. (2019) employed CA with multi-layer perceptron neural network to predict the demand for diary products in Iran. Selvarajah et al. (2019) applied CA to analysis the similarities between the users in online marketing. The results showed that CA is able to provide more accurate similarity analysis than GA. Sun et al. (2009) proposed a cultural-SA algorithm to solve the quality of service routing problem. Mohammadhosseini et al. (2019) proposed a virtual machine placement method based on CA to reduce the energy consumption in cloud data centres. Authors reported the efficiency of CA in comparison to a set of stochastic methods in the field of virtual machine placement.

Cowan, Reynolds (1999) applied CA to asses the quality of GP solution programs. Ostrowski, Reynolds (1999) suggested CA to solve the software testing problem, in which the white and black box testing methods were embedded into CA. Reynolds, Rolnick (1995); Reynolds, Rolnick (1995) proposed a image segmentation method based on CAs, in which a set of objects in a digital image are separated from their background. Authors used CAs to support the gradient-based method by finding its parameters.

\subsection{Other applications}

In the reviewed literature, there are some applications of CA in other research areas. Kobti et al. (2006a) utilised CA to study the emergence of a hub network from two kinship and economic networks, in which CA was used as a solution framework to embed the social intelligence in the system. Reynolds et al. (2008a) employed CA for mining the social fabric of archaic urban centres. Alexiou, Vlamos (2012) suggested CA for the representation of mitochondrial population in mammalian cells. Judeh et al. $(2014,2013)$ applied a gene set CA to reconstruct networks from unordered gene sets. Terán et al. (2017) utilised CA to optimise the coordination mechanisms in multi-agent 
Table 3: Hybrid versions of CA in literature.

\begin{tabular}{ll}
\hline Hybrid version & References \\
\hline CA+GA & Gao et al. (2006a), Gao et al. (2006b), Xue et al. (2011), Haikal, \\
CA+PSO & El-Hosseni (2011), Li et al. (2012), Tang, Li (2008) \\
& Lin et al. (2008), Sun et al. (2010), Zhang, Zhu (2012), Sun et al. \\
& (2012), Guo et al. (2017), Zhang et al. (2013), Lin et al. (2009), Chen \\
& et al. (2008), Wu et al. (2010),Santos Coelho dos, Mariani (2006), \\
& Ya-Li, Li-qing (2010), Stanley et al. (2019), Meng et al. (2011), Xie \\
& et al. (2009), Wang et al. (2017), Yu et al. (2011), Wei, Yan-Ping \\
& (2012), Guo et al. (2015), Wang et al. (2009), Chen et al. (2013) \\
& Arpaia et al. (2007), Niu et al. (2011), Becerra, Coello (2004), San- \\
CA+DE & tos Coelho dos et al. (2009), Tremayne et al. (2009), Dixit et al. \\
& (2019), Lu et al. (2011), Yuan et al. (2008), Khan et al. (2014), Khan \\
& et al. (2017), Awad et al. (2017), Ali et al. (2016b)m, Gao, Diao \\
& (2010), Yuan et al. (2009), Xu et al. (2012), Xu et al. (2010), Chen, \\
& Yang (2015) \\
& Ali, Reynolds (2014), De Freitas et al. (2018), Nguyen, Yao (2008), \\
& Nguyen, Yao (2006), Ali, Awad (2014), Ali et al. (2016a), Kim, Cho \\
& (2009), Awad et al. (2013), Sun et al. (2009), Ali et al. (2013),Di- \\
& galakis, Margaritis (2002), Jun et al. (2008) \\
& Gao et al. (2014), Guo et al. (2013b), Jafari et al. (2019), Gao, \\
& Diao (2011), Gao, Xu (2013), Guo et al. (2013a), Li et al. (2010), \\
& Gao et al. (2017a), Unold, Tarnawski (2016), Gao et al. (2019), \\
& Xiao et al. (2010), Pierezan et al. (2019), Murugadass, Sivakumar \\
& (2020), Xue (2020), Amalo et al. (2020), Hosseinioun et al. (2020), \\
& Santos Coelho dos, Alotto (2009) \\
&
\end{tabular}

systems in industrial automation. Pan et al. (2016) used CA with chaotic behaviour for cell allocation in CMOL circuits with the hybrid CMOS/nanodevice structure. Wei, Yan-Ping (2012) applied a CA-based PSO for partner selection of virtual enterprise and minimise time and cost.

\section{Variants of CAs}

Literature review shows that significant efforts have been done to increase the efficiency of CAs. As results of these efforts, different variants of CAs have been developed, ranging from improved and multi-population versions to hybridised versions and multi-objective versions. In this section, different variants of CAs developed in literature will be reviewed.

\subsection{Hybridisation of CAs}

Literature survey shows that CAs have been hybridised with other meta-heuristic techniques to increase their efficiency and robustness. Table 3 lists the hybridised versions of CAs with other meta-heuristic techniques, such as GAs, PSO, DE, and local search methods. In the following subsections, different versions of these hybridised schemes of CAs will be reviewed. 


\subsubsection{Hybrid CA and GA}

GA is a basic evolutionary algorithm inspired by the Darwinian theory of evolution. Literature review reveals that researchers have been hybridised CAs with GA to solve various optimisation problems. In most of the cases, they used GA as the evolutionary strategy to improve the fitness of individuals within the population space, while the knowledge components of the belief space of CAs are used as auxiliary tools to guide the operators of GA method.

Gao et al. (2006a) proposed an integrated CA and GA (CGA) algorithm for constrained optimisation. In CGA, an intelligent mutation operator was proposed to guide the search process towards the feasible solutions, in which the topographical knowledge component of belief space consisting of constraint characteristics of different regions of solution space was used as an auxiliary tool. From the reported results, it seems that CGA performed better than the standard GA and real coded GA in terms of solution quality and required computing time. A similar approach was also used by Gao et al. (2006b) to develop a hybrid model of CA and GA for optimisation problems. Xue et al. (2011) suggested a hybrid version of CA and GA, called NSCA, by introducing a novel selection operator in order to increase the population diversity. The approach used GA operators and knowledge components of belief space to generate new individuals. The results obtained for a nonlinear function revealed the superiority of NSCA over the standard CA.

Li et al. (2012) employed a hybrid cultural-GA to solve multi-aircraft cooperative target allocation problem. In cultural GA, the GA operators is used to evolve the population space, while the information of the belief space is employed to influence the selection and mutation operators. The results revealed that the cultural GA performs better than the standard GA. A similar hybrid cultural real-coded GA was developed by Haikal, El-Hosseni (2011) for constrained optimisation problems, in which the information about the higher quality solutions and the ranges of feasible regions are stored in the belief space to generate random offspring solutions around them. Authors compared the effectiveness of their approach against other hybrid algorithms, including gaussian PSO and quantum-behaved PSO. However, the comparisons between their approach and standard CA or GA were not provided. Tang, Li (2008) proposed a triple spaces cultured GA (TSCGA), in which a new space, called anti-culture population, was introduced to prevent premature convergence. In TSCGA, the anti-culture population space tries to generate new individuals outside the current cultural intervals, which can be helpful when the algorithm traps into the local optimum points.

\subsubsection{Hybrid CA and PSO}

As SI technique, PSO has attracted much attention as a suitable option to develop hybridised versions of CAs. Zhang, Zhu (2012) developed a cultural PSO to solve the JSP. In this algorithm, the adaptive PSO strategy is used to evolve the population space. It seems that authors tried to increase diversity and prevent premature convergence by replacing the global best by some of the poor solutions stored in belief space. Comparative results indicated that the cultural PSO was able to show better performance for JSPs than the standard PSO and its hybridised version. Lin et al. (2008) introduced a hybrid cultural cooperative PSO (CCPSO) for functional-link-based neural fuzzy network model in predictive applications. As illustrated in Fig. 3, CCPSO uses multiple swarms of particles with independent belief spaces, in which the positions of particles 
in each swarm are updated based on the knowledge components of the belief space corresponding to the same swarm. Reported convergence diagrams in this reference illustrate that the CCPSO performs remarkably better than the PSO and CPSO algorithms in terms of the convergence speed and solution quality. Similar approaches were also adopted by Lin et al. (2009); Chen et al. (2008). In another study, Sun et al. $(2010,2012)$ suggested a co-evolutionary cultural based PSO (CECBPSO), in which two sub-populations with different sub-belief spaces perform the optimisation process separately. In CECBPSO, the information collected within the two sub-belief spaces are shared with the global belief space. Then, the global belief space influences both of the population spaces. The general framework of the CECBPSO is given in Fig. 4. From the reported numerical results, it can be seen that the CECBPSO can perform significantly better than the standard PSO and its cooperative versions, such as CPSO.

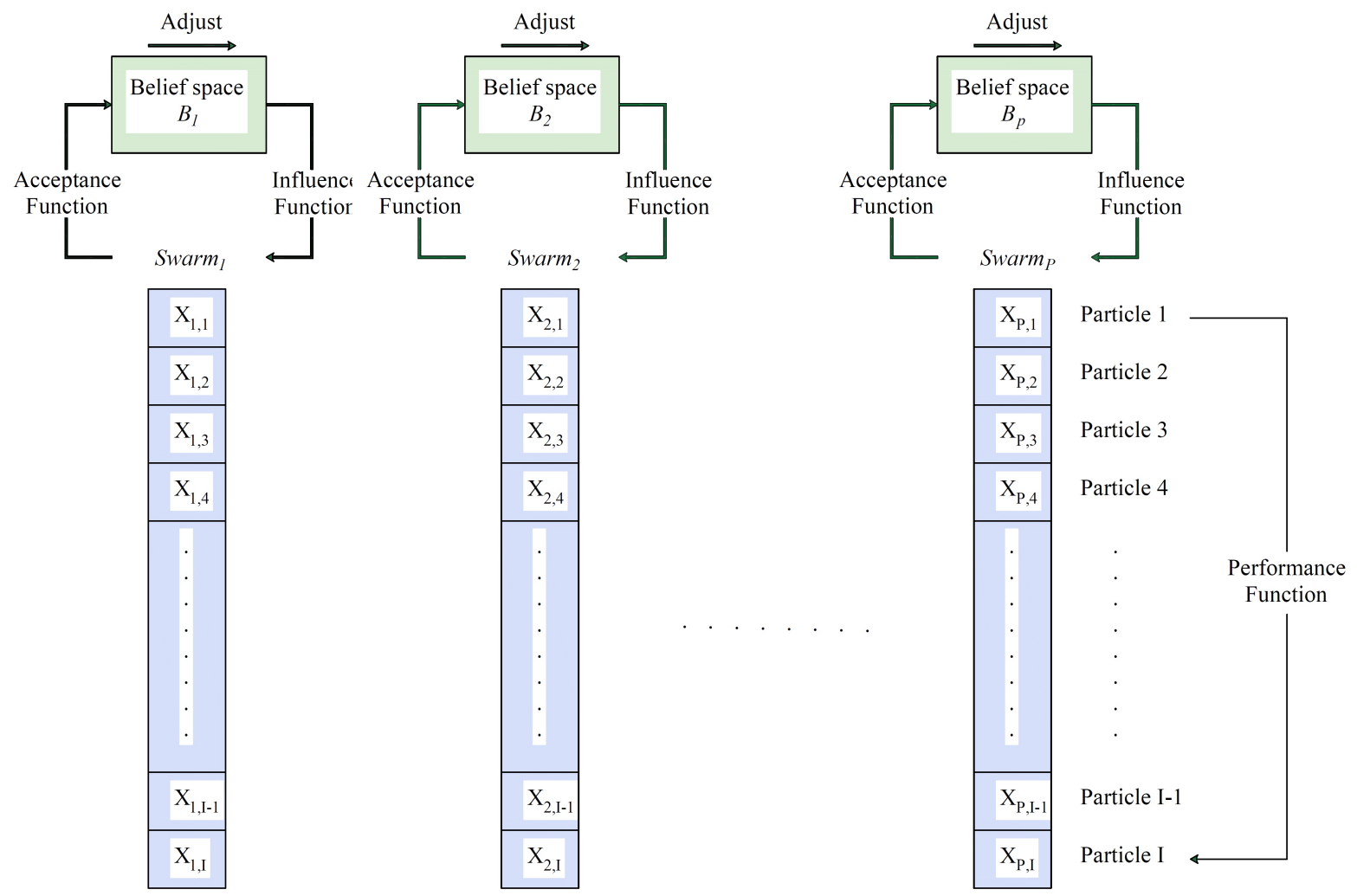

Figure 3: Framework of the hybrid CCPSO algorithm (Lin et al., 2008).

Guo et al. (2017) proposed cultural PSO algorithm for uncertain multi-objective problems with interval parameters, in which a possibility degree is used to construct a novel dominant comparison relationship. In this approach, the population space of the algorithm is evolved by the PSO algorithm, the flight parameters of PSO are adjusted by considering knowledge components, and the uniformity of non-dominant solutions in objective space is recorded in the topological knowledge component. Zhang et al. (2013) proposed an enhanced multi-objective CA algorithm (EMOCA), which combines PSO and CA framework. In this approach, the population is evolved by PSO, while the situational and history knowledge components are used to improve the convergence 


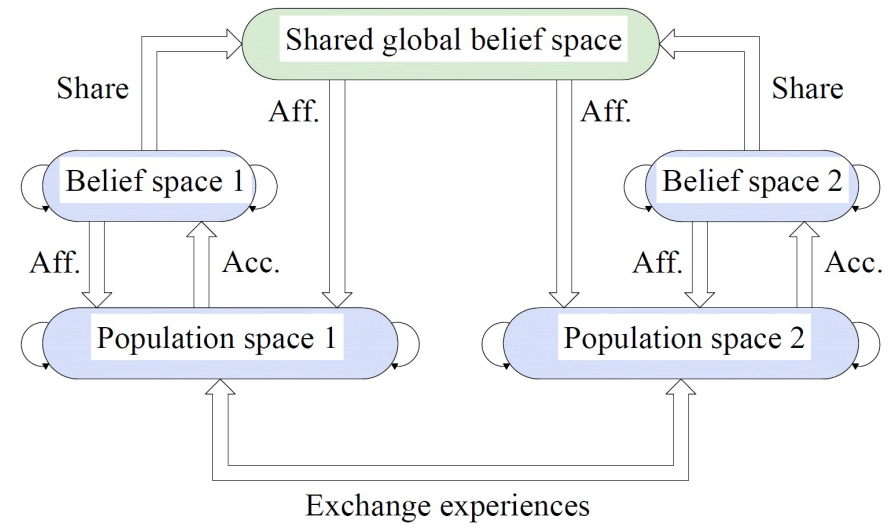

Figure 4: Framework of the hybrid CECBPSO algorithm (Sun et al., 2012, 2010).

speed. From reported Pareto fronts in this reference, it seems that EMOCA can provide more uniform distribution of non-dominate solutions in comparison to NSGAII and multi-objective DE algorithms.

Wu et al. (2010) suggested a hybrid CA and PSO (PSOCCA), in which the population space is evolved based on the modified PSO operators. In PSOCCA, different formulas based on the situational and normative knowledge components of belief space were proposed to update the positions of the particle. Convergence diagrams reported by authors shows that PSOCCA can provide better solutions with lower convergence speed than the standard PSO. This means the applicability of PSOCCA for problems with complex objective functions is limited. Santos Coelho dos, Mariani (2006) proposed a cultural Gaussian PSO (GPSO-CA) algorithm, in which the population space is evolved by the PSO algorithm with Gaussian random numbers. In GPSO-CA, the normative knowledge components of belief space are used to decide whether a variable should be updated.

Stanley et al. (2019) suggested a parallel multi-objective CA and PSO (CAPSO), in which several sub-swarms exchange the cultural information. In CAPSO, each subswarm searches a specific part of search space. Authors also investigated the effect of each knowledge component on the search process. Xie et al. (2009) presented a PSO algorithm based on CA (PSO-CA), in which the population space is evolved by PSO operators considering the normative knowledge component. An adaptive CA with IQPSO (ACA-IQPSO) was developed by Wang et al. (2017), in which IQPSO algorithm and CA are cooperating each other to generate new solutions. Authors proposed a new strategy to update the belief space and redesigned the acceptance and influence functions to enhance the utilisation of cultural information within the population. $\mathrm{Yu}$ et al. (2011) developed a HICHA for solving multi-robot exploration mission planning problem, in which the population space is evolved based on the PSO formulation. The general framework of HICHA is very similar to CA, in which the population and evolutionary spaces exchange information through a customer interactive estimation unit. 


\subsubsection{Hybrid CA and DE}

DE developed by Storn, Price (1997) is another population-based meta-heuristic algorithm which uses a set of search operators based on the weighted difference of solution vectors in search space. Arpaia et al. (2007) replaced the influence functions of CA by the mutation operators of DE algorithm to improve the performance of the standard $\mathrm{CA}$. Authors used the normative knowledge components to change the search direction, while the DE operators were used in the algorithm to generate new individuals. The new algorithm was performed better than GA. In another research, Niu et al. (2011) introduced a DE-based CA approach (CADE) to solve the hybrid JSPs. In CADE, the generated new solution vectors by normative-based influence function are randomly updated by DE operator. From the results reported by authors it can be seen that the CADE is statistically more efficient than the standard CA, PSO, and QEA. However, CADE requires slightly more computational effort in comparison to the standard CA. Similar approaches were also proposed by Khan et al. (2017) and Tremayne et al. (2009).

Becerra, Coello (2004) proposed a set of new influence functions for various knowledge components based on the DE strategies. In their approach, each DE-based influence function has a chance to update the position of each individual with a given probability. Similarly, Khan et al. (2014) suggested another hybrid version of CA and DE. By using the situational and normative knowledge components, authors proposed two new influence functions based on DE operators. These new influence functions combine three randomly selected individuals to form new generation of individuals in population space. Santos Coelho dos et al. (2009) presented an improved DE approach based on CA and measure of population's diversity (CDEMD) to solve EDP. In CDEMD, the positions of the individuals are updated using the mutation operators of DE defined based on the normative and situational knowledge components. Comparing to different versions of DE and PSO algorithms, it seems that the performance of CDEMD is remarkable.

Dixit et al. (2019) and Awad et al. (2017) suggested hybrid CA and DE algorithms, in which a participation ratio is introduced for each of CA and DE strategies. In the mentioned studies, the participation factor is updated during the optimisation process. The comparison results showed that the hybrid CA and DE approaches can perform significantly better than the different versions of CA and DE methods. Ali et al. (2016b) developed a hybrid cultural learning theme with a balanced performance for DE frameworks (b-hCA-DE). In b-hCA-DE, the population space is evolved by using the DE operators and the knowledge sources of the belief space, in which the role of each knowledge component is changed over time based on its success in generating high-quality solutions in the previous generations.

Lu et al. (2011) proposed a hybrid multi-objective CA (HMOCA) method, which combines the advantages of $\mathrm{CA}$ and $\mathrm{DE}$ to solve short-term environmental/economic hydrothermal scheduling problem. In HMOCA, the population space is evolved by the mutation operator of DE algorithm. The results showed that HMOCA is able to provide better distribution of Pareto front solutions than the NSGA-II method. Gao, Diao (2010) developed a differential cultural (DC) algorithm based on the differential-based influence functions. Convergence properties of DC are significantly better than those exhibited by PSO and its variants. Yuan et al. (2008) and Yuan et al. (2009) embedded DE into CA by using the situational and normative knowledge components within the variation operators. Authors used the selection operator of DE to distinguish between 
the feasible and infeasible solutions and handle the constraints of the problem. Comparisons showed that the hybrid approach performs better than DE and GA methods in terms of solution quality and required computational effort.

\subsubsection{Hybridisation with local search}

In some cases, researchers have been hybridised CAs with the local search techniques to improve the exploitation capability. TS and SA algorithms are known examples of such local search techniques. Ali, Reynolds (2014) suggested to use TS algorithm as the local optimiser to search the neighborhood of the optimal solutions obtained by CA in each generation. It seems that the hybrid algorithm performs statistically better than the standard CA and TS. Ali et al. (2013) hybridised the niche CA with TS algorithm. In their hybrid approach, the low-quality solutions are removed before performing local search by TS. A similar approach can be seen in (Ali, Awad, 2014). Sun et al. (2009) developed a cultural-SA (CA-SA) algorithm, in which the step size of the influence function of CA is control by the SA algorithm. Authors also used the temperature parameter of SA to update the situational knowledge of the belief space. The results revealed that CA-SA performs better than GA. However, statistical evidences for effectiveness of CA-SA against GA are not observable in the study. De Freitas et al. (2018) investigated the hybridisation of CA with SA and TS local search techniques for solving EDP. The convergence histories showed that the hybrid CA+TS was yielded significantly better results than $\mathrm{CA}+\mathrm{SA}$ hybridisation scheme. It can be seen from the convergence histories reported in this reference that the hybridisation scheme of CA+SA has not performed better than the standard CA.

Nguyen, Yao $(2006,2008)$ hybridised CA with the iterated local search to enhance the efficiency of the standard CA method. Ali et al. (2016a) developed a hybrid CA and modified multiple trajectory search (CA-MMTS) algorithm to improve the exploration and exploitation capabilities of CA. In CA-MMTS, the modified multiple trajectory search plays the role of local search strategy, which uses the knowledge components of belief space to enhance the quality of best solutions obtained by CA. Awad et al. (2013) proposed a hybrid CA and an improved local search (CA-ImLS) approach, in which the improved sub-local search is performed for each knowledge component of belief space. Jun et al. (2008) developed a hybrid CA-SA algorithm, in which the acceptance function works based on the SA formula. The convergence diagrams showed that the hybrid approach can find better solutions with fewer function evaluations than GA ans PSO methods.

\subsubsection{Other hybridisation schemes}

In recent years, a significant number of new meta-heuristic algorithms have been developed by researchers. Literature survey demonstrates that some studies have investigated the hybridisation schemes of CAs with these new approaches. Gao et al. (2014) introduced a quantum-inspired cultural BFA (QCBFA) by combining the quantum knowledge strategy and BFA, in which a quantum belief space is used to provide a guided cultural behaviour. Guo et al. (2013b) proposed a quantum-inspired CA (QCA) optimisation strategy to properly distribute sensor nodes in wireless sensor networks. In QCA, the population space is evolved by the QEA, and the implicit knowledge extracted from the high-quality solutions stored in belief space is used to update the positions of quantum individuals. Results showed that QCA performs better than both of 
the standard CA and QEA. A similar study was also performed by Guo et al. (2013a). Following the same idea, Guo et al. (2018) suggested interval multi-objective quantuminspired CA (IMOQCA). In this approach, the population space is evolved by the operators of the QEA, and the knowledge components of the belief space is used to guide the selection and mutation operations of evolutionary individuals. In another study, Gao, Xu (2013) proposed a cultural quantum-inspired SFLA for continuous optimisation. The algorithm uses the quantum knowledge strategy and new quantum leaping equations to enhance the performance of SFLA. As a cultural component, authors constructed a quantum belief space consisting of normative and situational knowledge components to model the cultural behaviour in the leaping movement.

Gao, Diao (2011) suggested a hybrid cultural firework (CF) algorithm based on the combination of firework algorithm and CA to deal with the filter design problem. Authors applied firework algorithm within the population space of CA, and added the cultural knowledge sources to the variation operator of firework evolution. Authors reported the efficiency of CF algorithm against PSO, quantum PSO, and adaptive quantum PSO algorithms. Santos Coelho dos, Alotto (2009) developed a cultural SOMA method for electromagnetic optimisation, in which the search direction in SOMA is obtained by considering the normative knowledge components of belief space. Comparison results showed that the cultural SOMA performs better than the classical SOMA and PSO methods. Jafari et al. (2019) suggested a hybrid EHO and CA (EHOC), in which the belief space of CA is used to improve the efficiency of EHO algorithm. EHO is a meta-heuristic algorithm developed by Wang et al. (2016), which mimics the herding behaviour of elephant groups. In EHOC, the cultural information of high-quality solutions stored in belief space are used to generate new solutions. The convergence diagrams reported in this reference show that the convergence speed of EHOC is faster than the standard CA and EHO methods. Murugadass, Sivakumar (2020) developed a hybrid EHO and CA (HEHO-CA) algorithm for optimal cluster head selection to extend the lifetime in wireless sensor networks. In HEHO-CA, it seems that the worst solutions in the population are replaced by the normative knowledge components in order to provide more accurate exploitation, while the situational knowledge component is used to improve the exploration ability.

Unold, Tarnawski (2016) introduced a paralel cultural ACO (pCACO) to solve the travelling salesman problem, in which CA and ACO-based max-min ant system algorithm are combined. In pCACO, the population space is evolved by ACO algorithm, while the belief space is evolved by a simple GA. In pCACO, the belief space of CA uses the created routes by the ants and tries to find the better ones. Xiao et al. (2010) introduced a cultural based ACO (ACCA), in which the new solutions generated by ACO are re-optimised by CA in each iteration. The results indicated that the ACCA approach has a better performance than GA. However, a fair statistical performance comparison was not carried out by the authors. Gao et al. (2019) developed a hybrid cultural HS algorithm, in which the knowledge components of CA is used to modify the harmonies generated by the HS algorithm. The convergence diagrams reported in this reference suggest that CA can significantly enhance the convergence rate of the HS algorithm. Gao et al. (2017a) suggested a hybrid CA and IWO algorithm (CIWA) to solve the design problem of digital watermarking with wavelet transform. In CIWA, the evolution of the population space is randomly performed by using cultural influence function and IWO operators. In a similar study, Hosseinioun et al. (2020) proposed a hybrid IWO and CA algorithm, in which the population space of CA is evolved by 
the operators of IWO algorithm.

Pierezan et al. (2019) proposed a cultural COA (CCOA), in which the normative knowledge of $\mathrm{CA}$ is used to improve the balance between the exploration and exploitation abilities of COA. Authors used the CA's normative-based influence function (similar to equation(12)) to update the solutions generated by COA. The numerical results obtained for the benchmark functions and gas demand minimisation of turbines reported in (Pierezan et al., 2019) revealed the normative-based influence function can significantly improve the solution qualities generated by the standard COA method. Guo et al. (2010) combined CA with $(\mu+\lambda)$ and clonal selection strategies, in which the influence of each selection method is controlled by the implicit knowledge resources. In another study, Mao et al. (2020) hybridised the FECO algorithm and CA for multiobjective numerical optimisation, in which the solutions generated by FECO are updated by randomly using knowledge components of CA. It seems that the hybrid algorithm has different internal parameters, which can affect the optimisation performance. Xue (2020) suggested an adaptive CA-based CS (CACS) algorithm, in which the solutions generated by CS algorithm are updated by CA. Authors reported that the hybrid CACS algorithm can provide better results than CA, CS, and ACO methods. Amalo et al. (2020) introduced a hybrid CA and BA to design a maximum power point tracking for a photo voltaic system, in which the normative knowledge component of CA is used to improve the convergence of BA.

\subsection{Communication topology for CAs}

One of the important factors that can affect the convergence properties of CAs is the efficiency of the knowledge distribution between the individuals within the population space. A recent survey revealed that the population structures with neighborhood or topological properties can significantly enhance the diversity preservation capacity of the population-based meta-heuristic algorithms (Lynn et al., 2018). Hence, researchers have been tried to control the knowledge dissemination through defining communication topologies within CAs (Ali, 2008; Che et al., 2010; Ali, Reynolds, 2009; King et al., 2008; Reynolds, Ali, 2008b,c,a). Ali, Reynolds (2014) employed simple forms of social network topologies to provide efficient knowledge dissemination and accelerate the convergence speed of CA, including ring, square, and global gBest topologies. Their approach benefits from the TS local search mechanism. The results obtained for function optimisation revealed the effectiveness of square topology. Ali et al. (2011a) proposed a new influence function based on the social fabric metaphor, in which the individuals in the populations are connected to each other by using ring (lBest) and global (gBest) topologies. Using these connections, individuals are able to share their experience with neighbourhood individuals. Authors reported that the lBest topology provides better results than $g B e s t$ topology. In another study, Ali et al. (2012) proposed a similar approach based on the ring (lBest), global (gBest), tree, and hybrid tree topologies. The statistical results reported in this reference show that the CA with social fabric influence function performs better than the standard CA and GA.

Reynolds, Gawasmeh (2012) and Ali et al. (2011b) investigated the performance of different homogeneous social networks, such as ring, square and global topologies, to properly spread the useful information among the individuals. From the reported results in these references, it seems that the impacts of communication topologies on CA's performance are more obvious in dealing with complex problems. Ali 
et al. (2015) developed a tribal sociocultural algorithm with neighbourhood restructuring (T-SCANeR) approach, in which the population is divided into multiple tribes with a given number of individuals for each. For each tribe, a given topology is defined, which shapes its social fabric structure and determines the interactions of individuals. Authors used different topologies to define the connection of individuals within tribes, including the ring, flattened representation of Von Neumann neighbourhood, hybrid-tree, and global topologies. In another study, Reynolds et al. (2015) introduced a sub-cultured topology concept in CAs based on a set of six homogenous topologies, in which the individuals can change their topologies during the evolution process. From the reported graphs in this reference, it can be seen that the sub-cultured concept is computationally more efficient than the homogeneous and heterogeneous approaches for complex problems. It seems that the sub-culture concept explores the search space with small groups of individuals, which is a positive feature from the exploration point of view.

\subsection{Improved CA}

Review of the literature revealed that several modifications have been made to improve the performance of $\mathrm{CA}$ in solving various optimisation problems. In this section, we outline some of the improved versions of CA.

Ali et al. (2014a) enhanced the balance between the exploration and exploitation of CAs through dynamic adjustment of the number of evaluations available for each type of knowledge source. In addition, authors used a local search to improve the solutions provided by the knowledge sources. Jalili et al. (2019) introduced an improved CA (ICA) for structural optimisation by using a new influence function to update the positions of individuals. Authors also used a truncated geometric distribution to simulate the number of dimension changes in each individual. The new influence function uses the normative and situational knowledge components simultaneously. The results showed that the ICA was able to perform significantly better than different versions of CA. Soza et al. (2007) integrated CA with variation operators to improve the exploration ability of the algorithm in solving timetabling problem. The algorithm proposed by the authors uses three cultural variation operators, including interchange, sequencing, and simple mutation operators. Haldar, Chakraborty $(2014,2015)$ suggested a modified CA (MCA) algorithm, in which the addition, multiplication, replacement and unchanged operators were used as the influence functions. Although the results confirmed that MCA performed better than the standard CA, some abrupt oscillations in convergence diagrams of MCA show that more examinations on the stability of the algorithm are required. Hence, a statistical analysis seems to be necessary to provide a fair comparison between CA and MCA.

Wang et al. (2015) proposed an improved CA for optimal vibration control of a vehicle's active suspension, in which the performance of the standard CA is enhanced by using the niche algorithm. In the improved version of CA, authors used niche algorithm within the acceptance function to accept the changeable number of individuals for updating the belief space. In their approach, the number of accepted solutions is gradually decreased over time. Al-Tirawi, Reynolds (2018) added a common value auction concept to the framework of CA in order to enhance the knowledge distribution between the individuals. The common value auction concept provides a set of parameters for each knowledge component of belief space, which include some infor- 
mation about each individual within the social network. Zhou et al. (2019) presented a cultural cognitive evolution optimisation (CCEO) algorithm, in which the cognitive behaviour is added to the CA framework to enhance the exploration and exploitation capabilities. CCEO is a three layer optimisation algorithm consisting of the population, belief, and cognitive spaces. In the cognitive space, the population is evolved by using the Gaussian random walk or Levy flight methods.

Bhattacharya et al. (2012b) suggested to use CA with single-point crossover to enhance the global search capability of CA. In comparison to other methods, the results obtained from a IEEE 14-bus system demonstrated that the approach was able to show comparable performance in terms of real power losses. Authors used a simple normal distribution as a mutation operator to perturb the solutions and increase the diversity. The sudden fluctuation in convergence diagram of the algorithm demands further statistical analysis. Reynolds, Kinnaird-Heether (2013) employed game theoretic mechanisms in order to make the role of the knowledge components more significant, including auction and weighted majority mechanisms. Based on the weighted majority win situation, the knowledge resources are able to predict the future success, while the auction mechanism allows the knowledge source to pro-actively compete for the individual based upon tokens derived from its current performances (Reynolds, Kinnaird-Heether, 2013). Mojab et al. (2019) proposed an improved CA by introducing a comprehensive elite mechanism, in which the information obtained from different knowledge components of the belief space is used for generating new solutions. The results revealed that not only improved CA performed better than the standard version of CA, but also exhibited better performance than other well-known meta-heuristic algorithms, such as GA and PSO. Waris, Reynolds (2018) improved the convergence properties of CA by using a new knowledge distribution mechanism based on the gametheoretic, in which the cooperation and competition between players were simulated.

\subsection{Multi-objective CA}

Literature review reveals that researchers have been adapted CA to solve multiobjective optimisation problems. Best et al. (2010) extended CA to solve multi-objective problems, in which they have modified different knowledge components of belief space to consider the effects of multiple objective functions. Srinivasan, Ramakrishnan (2012) introduced an extended version of CA for multi objective optimisation of classification rules, in which the individuals are accepted into the belief space based on the Pareto optimisation strategy using the metrics stored in the domain knowledge as vectors. Srinivasan, Ramakrishnan $(2013 \mathrm{~b}, \mathrm{a})$ extended CA to solve the multi-objective optimisation of classification rules. In the extended version of $\mathrm{CA}$, the individuals are categorised into imitator, cautious, and risk taker groups. Then, the new generation of solutions is produced by considering these traits. Cabrera et al. (2011) presented a bio-objective version of CA (BOCA), in which the $S$ metric was used as a metric for comparing two non-dominated sets. In BOCA, a given formula is also used to evaluate the fitness function of each individual.

Lagos et al. (2014) proposed a bi-objective CA (BOCA) to solve uncapacitated facility location problem and compared the effect of considering different knowledge components in the belief space. The results reported in this reference shows that the BOCA with history knowledge performs significantly better than the BOCA with normative and situational knowledge components. Guo et al. (2008) employed CA for 
multi-objective optimisation, in which the distributions of non-dominated individuals in objective space were stored in the implicit knowledge component. $\mathrm{Gu}, \mathrm{Wu}(2010)$ adapted CA to solve multi-objective problem of water resources optimisation, in which a non-dominate set is added to the general framework of CA. Coello, Becerra (2003) proposed to use CA with Pareto-based selection for multi-objective optimisation problems. Authors reported the competitive performance of CA in comparison to NSGA-II.

\subsection{Multi-population CA}

Researchers have been tried to develop multi-population versions of CAs. In some cases, the multi-population versions of CAs have been hybridised with other algorithms as well. For example, Gao et al. (2007) developed cultural particle swarm algorithms (CPSAs) by combining CA with PSO algorithm for constrained multi-objective optimisation. In CPSA algorithm, the population space is divided into $n+1$ subswarms, in which $n$ sub-swarms optimise the $n$ single objective functions separately, and one sub-swarm searches for the solution of multi-objective problem. Then, the knowledge obtained from each sub-swarm are used to update the belief space. Another multi-population version of CA was suggested by Zadeh, Kobti (2015), in which the solutions were divided into several sub-populations with a single belief space. The comparisons results revealed that the approach can provide better results than GA and DE. The general framework of their algorithm is very similar to the hybrid CPSA algorithm developed by Gao et al. (2007).

In another study, Yang, Gu (2014) presented a quadspace cultural genetic tabu algorithm (QSCGTA) to solve the JSP. In QSCGTA, the population space of CA is divided into junior and senior sub-populations with separate belief spaces. In QSCGTA, the junior population space is evolved by GA operators, while the senior population space is formed by TS algorithm. The reported graphs by authors show that the QSCGTA requires less computational time in comparison to NSGA-II and multi-objective version of PSO method. However, QSCGTA seems to be more complicated than the standard CA for implementation. Xu et al. (2012) introduced a multi-population cultural DE (MCDE) algorithm. The MCDE is consisted of two population spaces with separate belief spaces, which are evolving based on the DE operators. In MCDE, these populations exchange the information stored in their belief spaces. A diversity preservation mechanism was also used to alleviate premature convergence. Authors compared the results obtained by the MCDE to those yielded by PSO and GA. However, it seems that further investigations need to be carried out to compare the computational efficiency of MCDE against the standard CA. Unold, Tarnawski (2017) proposed a novel parallel framework for multi-population CA (MPCA), in which each sub-population has a separate belief space. In MPCA, a communication mechanism is provided to exchange the information between the sub-populations. The framework provides the possibility of using any heuristic approach for evolving population and belief spaces. However, the difficulties in implementation cannot be ignored.

Guo et al. (2011b) developed another multi-population version of CA, named multi-population CA adopting knowledge migration (MCAKM), in which a knowledge migration concept is proposed to provide more efficient interaction and cheaper communication between the sub-populations. In MCAKM, the implicit knowledge extracted from the evolution process of each sub-population is shared with other subpopulations based on the knowledge migration. Similar concepts were also adopted by 
Kobti, others (2012) and Guo et al. (2009) in a multi-population CA (MP-CA). In MPCA, the sub-populations perform as parallel local CAs and the extracted knowledge by each sub-population is shared with others in the form of structured belief using the knowledge migration. From the results reported in these references, it seems that the MP-CA performs statistically better than the CA with single-population for JSPs. $\mathrm{Xu}$ et al. (2010) suggested a multi-population cultural DE (MCDE), in which a knowledge exchange mechanism is defined to improve the evolution process. It seems that authors used a mechanism to prevent cultural fusion and preserve the diversity, in which the main aim is to keep the populations away from each other in the search space.

Digalakis, Margaritis (2002) presented the parallel co-operating CA (PARCA) to solve the maintenance scheduling problem. In PARCA, several sub-populations investigate the different data set, in which the information exchange is performed by the accepted individuals from each sub-population. Authors added different local search mechanisms to PARCA. However, the advantages of PARCA over the standard CA were not investigated. Guo et al. (2011c) developed a multi-population cooperative CA (MCCA) with several sub-population spaces evolving with the competition cooperative GA. Authors verified the efficiency of MCCA against GA and cooperative GA. Kobti, others $(2014,2013)$ introduced a heterogeneous multi-population CA (HMP-CA) with a set of sub-populations communicated through a shared belief space. In HMP$\mathrm{CA}$, each sub-population optimises a given variable using DE mutation operator.

Ali, Awad (2014) suggested niche CA (NCA) method based on the parallel niche groups and niche clearing techniques, in which the population space is divided into several parallel sub-populations to perform local search separately. In each iteration, the poor solutions are removed from the population based on the niche-clearing technique. Yan et al. (2016) suggested another niching CA (NCA) with population space divided into several sub-populations. The approach modifies the size of each subpopulation based on the average fitness values. In this approach, the belief space is divided into several parts, in which the knowledge components related to each subpopulation are stored in a given part. In another study, Yan et al. (2017b) introduced an improved CA based on the niche isolation technology, in which each sub-population evolves independently. It seems that the general performance of the approach is significantly better than the standard CA and GA in terms of the solution quality and required computing time. A clan-based CA approach was developed by Oloruntoba et al. (2019) by defining multiple clans (or sub-populations) with independent local belief spaces. Authors defined a migration mechanism to exchange information between the clans. The algorithm has a global belief space to gather the information obtained from the different clans. From the results reported in this reference, it seems that the clan-based CA can provide more accurate results than PSO, ACO, and CS algorithms.

Reynolds, Kinnaird-Heether (2017a) developed a population mechanism as a new knowledge distribution method, which mimics the specialist or professional networks in real human societies. The population mechanism models several evolving networks between the individuals, which lead to development of several subcultures within the system. In another study, Gawasmeh, Reynolds (2014) investigated the impact of the sub-cultures on the performance of CAs, in which they concluded that the presence of sub-cultures can improve the computational efficiency. Singh et al. (2018) proposed a multi-population version of CA, in which the population is divided into several groups evolving separately. Then, some selection operators are used between the groups to generate new solutions. Chen, Yang (2015) developed a DE-based symbiotic CA 
(DESCA) to design a fuzzy system. In DESCA, each individual represents a fuzzy system consisting of a set of fuzzy rules. The algorithm divides the population space into several sub-populations, in which the individuals in each sub-population represent a given rule of fuzzy system. The new rules in DESCA are generated by using mutation strategies of DE algorithm and knowledge components of CA. It is observable from the convergence diagrams and results reported in this reference that DESCA performs significantly better than the different versions of DE algorithm.

From the literature review on the multi-population versions of $\mathrm{CA}$, it seems that the solution quality of CA can be enhanced by dividing its population. However, the main question in this area is how these techniques are computationally efficient than the standard CAs? It seems that this question has not been answered in the abovementioned references.

\subsection{Chaotic CA}

In recent years, the chaotic theory in nonlinear dynamics has attracted much attention from the researchers in the field of the meta-heuristic algorithms. Researchers have been used the chaotic sequences as pseudo-random sequences to improve the performance of meta-heuristic algorithms. There are some studies that the chaotic maps were integrated with CA to achieve better performance. For example, Wang et al. (2009) developed a chaos culture PSO (CCPSO) for function optimisation. In CCPSO, the population space is evolved by the PSO algorithm and a chaotic search based on the logistic chaotic map is performed within the belief space to prevent premature convergence. Pan et al. (2016) designed a CA with chaotic behaviour, in which the individuals are evolving within both of the population and belief spaces. Authors used GA to evolve the population space, while the individuals in the belief space are evolved by chaotic ACO algorithm. It seems that the approach performs computationally cheaper than conventional GA for cell allocation in CMOL circuits with the hybrid CMOS/nanodevice structure. Guo et al. (2011a) proposed a mixed mutation operators based on the Cauchy+chaotic and Cauchy+Gaussian mutation operators within the CA.

Cheng et al. (2009) suggested an adaptive chaotic CA by using a mutation based on the chaotic logistic map within the population space. Although the approach was able to provide accurate results, the effects of applying chaotic map on the performance of CA were not investigated. Chen et al. (2013) presented a cultural evolution algorithm, which uses PSO operators and chaotic maps to evolve the population and belief spaces. $\mathrm{He}, \mathrm{Xu}$ (2011) developed a chaotic CA (CCA), in which two chaotic-based influence functions using normative and situational knowledge components were proposed. In these chaotic influence functions, chaotic random variables were generated based on the logistic map function.

Although the literature review described in this subsection reveals that researchers have been tried to model the chaotic behaviour in CAs, the questions on the effects of chaotic sequences on the performance of CAs remain unaddressed. Furthermore, all of the above-mentioned references have been used the logistic map function to model chaotic behaviour in CAs. It seems that the suitability of other chaotic maps on the performance of CAs should be investigated as well, such as Logistic, Kent, Bernoulli shift, Sine, and Circle maps. 


\subsection{Fuzzy CA}

Literature review reveals that some researchers combined CA with fuzzy sets theory to speed up its convergence speed. For example, Reynolds, Zhu (2001) developed a fuzzy CA approach with fuzzy acceptance and influence functions, in which the knowledge components of the belief space were represented based on the fuzzy framework. Authors investigated different configurations of fuzzy components on the algorithm's performance. Ali et al. (2018) introduced a balanced fuzzy CA with a modified Levy flight search $(b-f C A+m L F)$ based on the modified fuzzy acceptance and influence functions. Authors designed the fuzzy acceptance function in a way to accept a large number of individuals in initial iterations, while this number is gradually reduced over time. In the influence functions, authors defined a fuzzy parameter to control the search direction. A similar approach was also suggested by Muhamediyeva (2020). Alami et al. (2007) developed a multi-population CA based on the fuzzy clustering, in which the fuzzy clustering is used to divide the population space into a set of sub-populations. Feng, Zhang (2008) proposed a self-adaptive CA based on the fuzzy controller to speed up the convergence of the standard CA. Authors suggested to define the number of accepted individuals as a fuzzy number. Reynolds, Chung (1997a) proposed a new acceptance function using a fuzzy inference engine to select the individuals from the population space. From the results reported in this reference, it can be seen that the CA with fuzzy acceptance function provides significantly better results than those provided by $\mathrm{CA}$ with static acceptance function.

\subsection{CAs with EP and GP}

Some studies have been done on combining CAs with EP and GP approaches. Coello Coello, Becerra (2004) applied CA to improve the performance of EP technique. The study uses the domain knowledge component of belief space to identify the feasible regions of search space and guide the search process. Coello, Becerra (2002b) proposed an approach based on CAs and EP for constrained optimisation. Chung, Reynolds (1996b) used CAs to provide the self-adaptation at the population level for EP systems. Comparisons of the convergence diagrams revealed that CA can significantly accelerate the convergence speed of EP. Huang et al. (2008) developed a CA with multi-layer belief spaces and EP. Ostrowski, Reynolds $(2004,2005)$ applied a chained CA with GP to evolve strategies for recessionary markets, in which two separate CAs were used for each of the black-box and white-box testings. Reynolds et al. (2008b) simulated the evolution of an ancient urban centre via embedding the GP within CA. Zannoni, Reynolds (1997) proposed a novel CA and GP (CAGP) computational system to solve symbolic regression problem, which is consisted of the population and belief components. In CAGP, the programs and genetic operators follow the constraints provided by the belief space. The convergence diagrams reported by authors illustrate the superiority of CAGP over pure GP approach.

\section{Publication analysis}

In this section, some information about the publications related to the CAs are provided. This survey paper considers around 245 references related to CAs and their variants in different publications, such as IEEE, Elsevier, Springer, Taylor Francis, and John Wiley. These references are consisting of books, journal and conference papers. 
Fig. 5 shows the chronological distribution of related publications to the CAs over the recent years. Fig. 5 illustrates that CAs have attracted significant attentions in the last decade. In addition, Fig. 6 illustrates the distribution of CAs related publications in different research areas. From this figure, it can be seen that CAs have been attracted much attention from the researchers in computer science and electrical engineering. Moreover, Fig. 7 presents the distribution of the investigated references in different publishers, including Springer, IEEE, Elsevier, and others. As it can be seen from Fig. 7 , most of these references are published by IEEE and Springer.

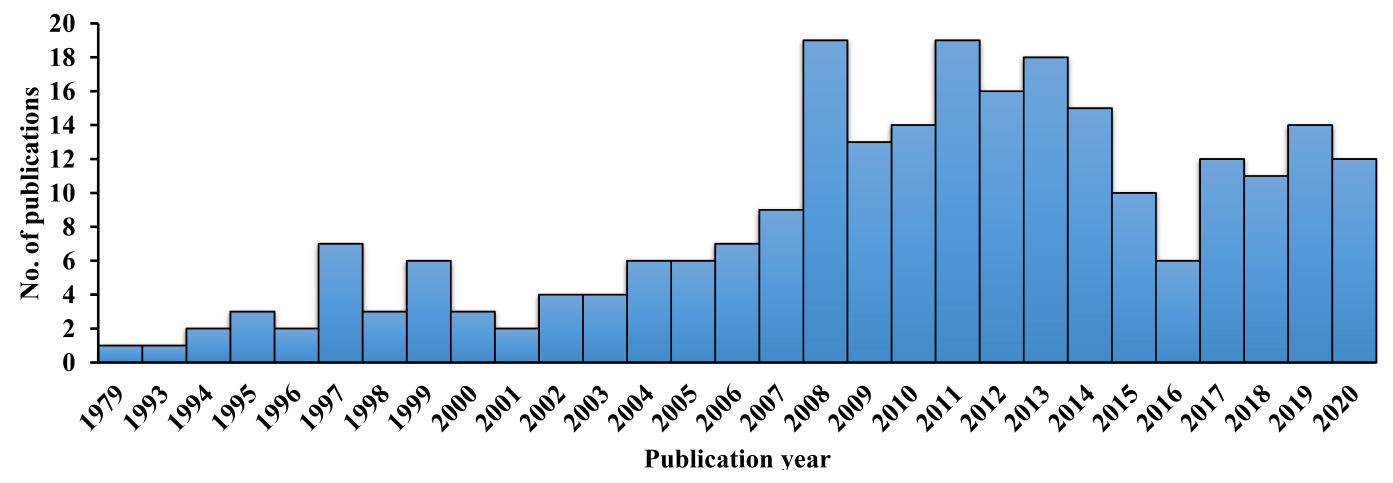

Figure 5: The number of publications related to CAs in recent years.

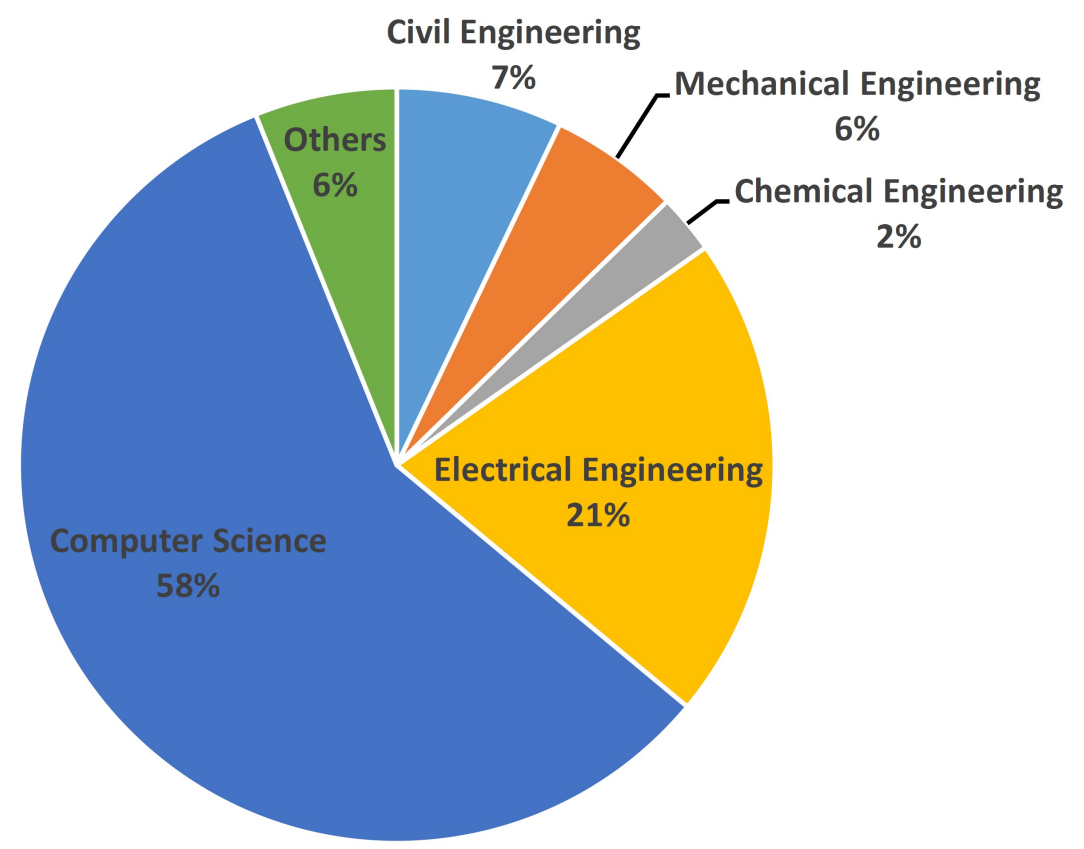

Figure 6: Distribution of CA related papers in different fields. 


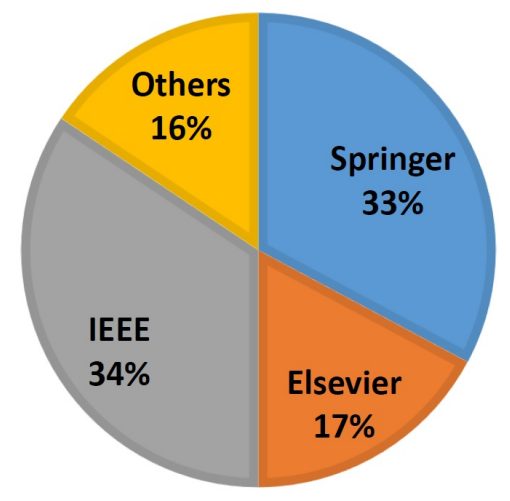

Figure 7: The references of CA in different publications.

\section{Conclusions and future research directions}

This paper attempted to provide a comprehensive survey of CAs and their applications in science and engineering as well as their development during the recent years. Regarding the applications, CAs have been successfully applied to solve a wide variety of optimisation problems in science and engineering, such as civil engineering, mechanical engineering, chemical engineering, electrical engineering and computer science. Literature review of most relevant references has demonstrated that CAs have been attracted much attention from the researchers in computer science and electrical engineering. The review of this body of literature has revealed that a significant number of variants of CAs have been developed by researchers, which can be categorised into hybridised, improved, multi-objective, multi-population, chaotic, and fuzzy variants. Although the literature review indicates the maturity of CAs as effective optimisation approach, there are significant issues remaining to be addressed in future works, as follows:

- In the last decade, numerous novel meta-heuristic algorithms have been introduced in literature. The review has reveled that most of the hybridised versions of CAs with other meta-heuristic algorithms have been focused on the hybridising CAs with GA, DE, and PSO approaches. Therefore, it may be of interest to investigate hybridisation possibilities of CAs with other recently developed metaheuristic algorithms. The capabilities of the belief space to provide multiple types of knowledge sources can be efficiently combined with other meta-heuristic techniques.

- Literature survey revealed an overall consensus that properly designed influence functions have the potential to use the information of the knowledge sources more efficiently. The key point in designing the influence functions is keeping the diversity of the population space to prevent premature convergence. Therefore, developing efficient influence functions to deal with the different problem environments seems to be vital, and future research should take a few steps forward to this direction.

- Another important component of CAs is the update function, which is used to update the information stored within the knowledge sources of the belief space. 
In some cases, the knowledge sources are failed to provide useful information for the population space, which leads to the premature convergence of the algorithm. For example, too narrow normative intervals can easily reduce the convergence speed of the algorithm. Thus, there is a need for future research on improving the update function to keep the cultural information useful during the solutionfinding process.

- Analysis of the literature demonstrated that researchers have been used different types of knowledge sources within the belief space to deal with different problems. However, the current state of research does not provide a clear analysis of the impact of various knowledge sources and their coordination on the performance of CAs. Hence, a rigorous study on the impacts of the knowledge sources in different problem environments seems to be necessary.

- Another important challenge is the adapting CAs to solve multi-objective optimisation problems. Literature survey has also revealed that CAs are put forward to solve multi-objective problems in different areas of science and engineering. However, even if research is successful in applying CAs to solve these types of problems, there will certainly be gaps that require research to address this challenge. Future research will definitely be required to design a unified framework for CAs to deal with these problems.

- Although CAs are capable of dealing with the dynamic optimisation problems, their performance on dynamic problems has been rarely investigated. Hence, the capabilities of CAs in solving the dynamic problems needs to addressed in future researches.

\section{References}

Abdolrazzagh-Nezhad Majid. Enhanced cultural algorithm to solve multi-objective attribute reduction based on rough set theory // Mathematics and Computers in Simulation. 2020. 170. 332-350.

Al-Tirawi Anas, Reynolds Robert G. Using Common Value Auction In Cultural Algorithm to Enhance Robustness and Resilience of Social Knowledge Distribution Systems // 2018 IEEE Symposium Series on Computational Intelligence (SSCI). 2018. 755-764.

Alami J, El Imrani A. Using cultural algorithm for the fixed-spectrum frequency assignment problem / Journal of Mobile Communication. 2008. 2, 1. 1-9.

Alami J, El Imrani A, Bouroumi Abdelaziz. A multipopulation cultural algorithm using fuzzy clustering // Applied Soft Computing. 2007. 7, 2. 506-519.

Alexiou Athanasios, Vlamos Panayiotis. A cultural algorithm for the representation of mitochondrial population // Advances in Artificial Intelligence. 2012. 2012. 1.

Ali Mostafa, Reynolds Robert, Ali Rose, Salhieh Ayad. Knowledge-based constrained function optimization using cultural algorithms with an enhanced social influence metaphor / / Computational Intelligence. 2011a. 103-119.

Ali Mostafa Z. Using cultural algorithms to solve optimization problems with a social fabric approach. 2008. 
Ali Mostafa Z, Awad Noor, Reynolds Robert G. Balancing search direction in cultural algorithm for enhanced global numerical optimization / / 2014 IEEE Symposium on Swarm Intelligence. 2014a. 1-7.

Ali Mostafa Z, Awad Noor H. A novel class of niche hybrid cultural algorithms for continuous engineering optimization // information sciences. 2014. 267. 158-190.

Ali Mostafa Z, Awad Noor H, Reynolds Robert G. Hybrid niche cultural algorithm for numerical global optimization // 2013 IEEE Congress on Evolutionary Computation. 2013. 309-316.

Ali Mostafa Z, Awad Noor H, Reynolds Robert G, Suganthan Ponnuthurai N. A balanced fuzzy cultural algorithm with a modified levy flight search for real parameter optimization // Information Sciences. 2018. 447. 12-35.

Ali Mostafa Z, Awad Noor H, Suganthan Ponnuthurai N, Duwairi Rehab M, Reynolds Robert G. A novel hybrid Cultural Algorithms framework with trajectory-based search for global numerical optimization // Information Sciences. 2016a. 334. 219249.

Ali Mostafa Z, Awad Noor H, Suganthan Ponnuthurai N, Reynolds Robert G. A modified cultural algorithm with a balanced performance for the differential evolution frameworks // Knowledge-Based Systems. 2016b. 111. 73-86.

Ali Mostafa Z, Morghem Abdulmalik, Albadarneh Jafar, Al-Gharaibeh Rami, Suganthan Ponnuthurai $N$, Reynolds Robert $G$. Cultural algorithms applied to the evolution of robotic soccer team tactics: a novel perspective / / 2014 IEEE congress on evolutionary computation (CEC). 2014b. 2180-2187.

Ali Mostafa Z, Reynolds Robert G. An intelligent social fabric influence component in cultural algorithms for knowledge learning in dynamic environments // 2009 IEEE/WIC/ACM International Joint Conference on Web Intelligence and Intelligent Agent Technology. 2. 2009. 161-168.

Ali Mostafa Z, Reynolds Robert G. Cultural algorithms: a Tabu search approach for the optimization of engineering design problems // Soft Computing. 2014. 18, 8. 16311644.

Ali Mostafa Z, Salhieh Ayad, Snanieh Randa T Abu, Reynolds Robert G. Boosting cultural algorithms with an incongruous layered social fabric influence function / / 2011 IEEE Congress of Evolutionary Computation (CEC). 2011b. 1225-1232.

Ali Mostafa Z, Salhieh Ayad, Snanieh Randa T Abu, Reynolds Robert G. Boosting cultural algorithms with a heterogeneous layered social fabric influence function // Computational and Mathematical Organization Theory. 2012. 18, 2. 193-210.

Ali Mostafa Z, Suganthan Ponnuthurai N, Reynolds Robert G, Al-Badarneh Amer F. Leveraged neighborhood restructuring in cultural algorithms for solving real-world numerical optimization problems // IEEE transactions on evolutionary computation. 2015. 20, 2. 218-231.

Amalo Kulu Amadu, Birninkudu Sabo Ibrahim, Bukata Bala Boyi, Salawudeen Ahmed Tijani, Ahmad Ashraf Adam. Cultured Bat Algorithm for Optimized MPPT Tracking Under Different Shading Conditions // 2020 International Conference in Mathematics, Computer Engineering and Computer Science (ICMCECS). 2020. 1-8. 
Arpaia Pasquale, Lucariello Giuseppe, Zanesco Antonio. Automatic fault isolation by cultural algorithms with differential influence / / IEEE Transactions on Instrumentation and Measurement. 2007. 56, 5. 1573-1582.

Awad Noor H, Ali Mostafa Z, Duwairi Rehab M. Cultural algorithm with improved local search for optimization problems // 2013 IEEE Congress on Evolutionary Computation. 2013. 284-291.

Awad Noor H, Ali Mostafa Z, Suganthan Ponnuthurai N, Reynolds Robert G. CADE: a hybridization of cultural algorithm and differential evolution for numerical optimization / / Information Sciences. 2017. 378. 215-241.

Becerra Ricardo Landa, Coello Carlos A Coello. A cultural algorithm with differential evolution to solve constrained optimization problems / / Ibero-American Conference on Artificial Intelligence. 2004. 881-890.

Becerra Ricardo Landa, Coello Carlos A Coello. A cultural algorithm for solving the job shop scheduling problem / / Knowledge incorporation in evolutionary computation. 2005. 37-55.

Best Christopher, Che Xiangdong, Reynolds Robert G, Liu Dapeng. Multi-objective cultural algorithms // IEEE Congress on Evolutionary Computation. 2010. 1-9.

Bhattacharya Bidishna, Mandal Kamal, Chakraborty Niladri. Knowledge Based Evolutionary Programming: Cultural Algorithm Approach for Constrained Optimization // Proceedings of the International Conference on Information Systems Design and Intelligent Applications 2012 (INDIA 2012) held in Visakhapatnam, India, January 2012. 2012a. 93-101.

Bhattacharya Bidishna, Mandal Kamal Krishna, Chakraborty Niladri. Reactive power optimization using hybrid cultural algorithm // International Conference on Swarm, Evolutionary, and Memetic Computing. 2012b. 106-115.

Cabrera Guillermo, Rubio José Miguel, Díaz Daniela, Fernández Boris, Cubillos Claudio, Soto Ricardo. A cultural algorithm applied in a bi-objective uncapacitated facility location problem / / International Conference on Evolutionary Multi-Criterion Optimization. 2011. 477-491.

Cai Zhaohui, Zhang Min, Liu Yujiao. Sea-surface weak target detection scheme using a cultural algorithm aided time-frequency fusion strategy // IET Radar, Sonar \& Navigation. 2018. 12, 7. 711-720.

Ceylan Huseyin, Ceylan Halim, Haldenbilen Soner, Baskan Ozgur. Transport energy modeling with meta-heuristic harmony search algorithm, an application to Turkey // Energy policy. 2008. 36, 7. 2527-2535.

Che Xiangdong, Ali Mustafa Z, Reynolds Robert Gene. Weaving the Social Fabric: The Past, Present, and Future of Optimization Problem Solving with Cultural Algorithms // 2010 AAAI Fall Symposium Series. 2010.

Chen Cheng-Hung, Liu Yong-Cheng, Lin Cheng-Jian, Lin Chin-Teng. A hybrid of cooperative particle swarm optimization and cultural algorithm for neural fuzzy networks / / 2008 IEEE International Conference on Fuzzy Systems (IEEE World Congress on Computational Intelligence). 2008. 238-245. 
Chen Cheng-Hung, Yang Sheng-Yen. Efficient DE-based symbiotic cultural algorithm for neuro-fuzzy system design // Applied Soft Computing. 2015. 34. 18-25.

Chen L, Pan ZL, Zhang GZ, Wu PH. A Novel Design Approach of High Temperature Superconducting Magnets by Cultural Evolution Algorithms // Journal of Low Temperature Physics. 2013. 170, 5-6. 359-365.

Cheng Jian, Qian Jiansheng, Guo Yi-nan. Adaptive chaotic cultural algorithm for hyperparameters selection of support vector regression // International Conference on Intelligent Computing. 2009. 286-293.

Chu PC, Beasley John E. Constraint handling in genetic algorithms: the set partitioning problem / / Journal of Heuristics. 1998. 4, 4. 323-357.

Chung Chan-Jin, Reynolds Robert G. A Testbed for Solving Optimization Problems Using Cultural Algorithms. // Evolutionary programming. 1996a. 225-236.

Chung Chan-Jin, Reynolds Robert G. CAEP: An evolution-based tool for real-valued function optimization using cultural algorithms // International Journal on Artificial Intelligence Tools. 1998. 7, 03. 239-291.

Chung ChanJin, Reynolds Robert G. Function optimization using evolutionary programming with self-adaptive cultural algorithms // Asia-Pacific Conference on Simulated Evolution and Learning. 1996b. 17-26.

Clayton Nicola S, Griffiths Daniel P, Dickinson Anthony. Declarative and episodic-like memory in animals: Personal musings of a Scrub Jay // The evolution of cognition. 2000. 273288.

Coello Carlos A Coello, Becerra Ricardo Landa. Adding knowledge and efficient data structures to evolutionary programming: A cultural algorithm for constrained optimization // Proceedings of the 4th Annual Conference on Genetic and Evolutionary Computation. 2002a. 201-209.

Coello Carlos A Coello, Becerra Ricardo Landa. A cultural algorithm for constrained optimization // Mexican International Conference on Artificial Intelligence. 2002b. 98-107.

Coello Carlos A Coello, Becerra Ricardo Landa. Evolutionary multiobjective optimization using a cultural algorithm / / Proceedings of the 2003 IEEE Swarm Intelligence Symposium. SIS'03 (Cat. No. 03EX706). 2003. 6-13.

Coello Coello Carlos A, Becerra Ricardo Landa. Efficient evolutionary optimization through the use of a cultural algorithm / / Engineering Optimization. 2004. 36, 2. 219-236.

Cortés Rivera Daniel, Landa Becerra Ricardo, Coello Coello Carlos A. Cultural algorithms, an alternative heuristic to solve the job shop scheduling problem // Engineering Optimization. 2007. 39, 1.69-85.

Cowan George S, Reynolds Robert G. Learning to assess the quality of genetic programs using cultural algorithms // Proceedings of the 1999 Congress on Evolutionary Computation-CEC99 (Cat. No. 99TH8406). 3. 1999. 1679-1686. 
Crawford Broderick, Lagos Carolina, Castro Carlos, Paredes Fernando. A cultural algorithm for solving the set covering problem // Analysis and Design of Intelligent Systems using Soft Computing Techniques. 2007. 408-415.

Crawford Broderick, Soto Ricardo, Monfroy Eric. Cultural algorithms for the set covering problem / / International Conference in Swarm Intelligence. 2013. 27-34.

De Freitas Carlos Alberto Oliveira, Oliveira Roberto Celio Limão de, Da Silva Deam James Azevedo, Leite Jandecy Cabral, Junior Jorge De Almeida Brito. Solution to Economic-Emission Load Dispatch by Cultural Algorithm Combined With Local Search: Case Study / / IEEE Access. 2018. 6. 64023-64040.

Del Ser Javier, Osaba Eneko, Molina Daniel, Yang Xin-She, Salcedo-Sanz Sancho, Camacho David, Das Swagatam, Suganthan Ponnuthurai N, Coello Carlos A Coello, Herrera Francisco. Bio-inspired computation: Where we stand and what's next // Swarm and Evolutionary Computation. 2019. 48. 220-250.

Artificial Immune Network Clustering Based on a Cultural Algorithm. // . 2020.

Digalakis Jason G, Margaritis Konstantinos G. A multipopulation cultural algorithm for the electrical generator scheduling problem / / Mathematics and Computers in Simulation. 2002. 60, 3-5. 293-301.

Dixit Abhishek, Kumar Sushil, Pant Millie, Bansal Rohit. CA-DE: Hybrid Algorithm Based on Cultural Algorithm and DE // Machine Intelligence and Signal Analysis. 2019. 185-196.

Dorigo Marco, Di Caro Gianni. Ant colony optimization: a new meta-heuristic // Proceedings of the 1999 congress on evolutionary computation-CEC99 (Cat. No. 99TH8406). 2. 1999. 1470-1477.

Eberhart Russell, Kennedy James. Particle swarm optimization // Proceedings of the IEEE international conference on neural networks. 4. 1995. 1942-1948.

Engelbrecht Andries P. Computational intelligence: an introduction. 2007.

Erol Osman K, Eksin Ibrahim. A new optimization method: big bang-big crunch // Advances in Engineering Software. 2006. 37, 2. 106-111.

Feng Wang, Zhang Xue-ying. The self-adaptive cultural algorithm optimization based on the fuzzy controller // 2008 IEEE International Symposium on Knowledge Acquisition and Modeling Workshop. 2008. 328-332.

Serious Game Modeling of Caribou Behavior Across Lake Huron Using Cultural Algorithms and Influence Maps. // . 2011.

Gallo Mariano, D'Acierno Luca, Montella Bruno. A meta-heuristic approach for solving the urban network design problem // European Journal of Operational Research. 2010. 201, 1. 144-157.

Gandomi Amir Hossein, Alavi Amir Hossein. Krill herd: a new bio-inspired optimization algorithm // Communications in nonlinear science and numerical simulation. 2012. 17, 12. 4831-4845. 
Gao Fang, Cui Gang, Liu Hongwei. Integration of genetic algorithm and cultural algorithms for constrained optimization // International Conference on Neural Information Processing. 2006a. 817-825.

Gao Fang, Liu Hongwei, Zhao Qiang, Cui Gang. Hybrid model of genetic algorithm and cultural algorithms for optimization problem // Asia-Pacific Conference on Simulated Evolution and Learning. 2006b. 441-448.

Gao Fang, Zhao Qiang, Liu Hongwei, Cui Gang. Cultural particle swarm algorithms for constrained multi-objective optimization // International Conference on Computational Science. 2007. 1021-1028.

Gao HY, Chi PF, Du YN, Wang Y, Diao M. Digital Watermarking Based on Wavelet Transform and Cultural Invasive Weed Algorithm // International Conference in Communications, Signal Processing, and Systems. 2017a. 1620-1627.

Gao Hongyuan, Diao Ming. Differential cultural algorithm for digital filters design // 2010 Second International Conference on Computer Modeling and Simulation. 3. 2010. 459-463.

Gao Hongyuan, Diao Ming. Cultural firework algorithm and its application for digital filters design / / International Journal of Modelling, Identification and Control. 2011. $14,4.324-331$.

Gao Hongyuan, Xu Congqiang. Cultural quantum-inspired shuffled frog leaping algorithm for direction finding of non-circular signals // International Journal of Computing Science and Mathematics. 2013. 4, 4. 321-331.

Gao Hongyuan, Xu Congqiang, Li Chenwan. Quantum-inspired cultural bacterial foraging algorithm for direction finding of impulse noise // International Journal of Innovative Computing and Applications. 2014. 6, 1. 44-54.

Gao Kaizhou, Zhang Yicheng, Sadollah Ali, Lentzakis Antonios, Su Rong. Jaya, harmony search and water cycle algorithms for solving large-scale real-life urban traffic light scheduling problem / / Swarm and evolutionary computation. 2017b. 37. 58-72.

Gao Min, Zhu Yanfei, Cao Cuiwen, Zhu Yanfeng. A Hybrid Cultural Harmony Search Algorithm for Constrained Optimization Problem of Diesel Blending // IEEE Access. 2019.

Gawasmeh Yousof A, Reynolds Robert G. A computational basis for the presence of subcultures in cultural algoithms / / 2014 IEEE Symposium on Swarm Intelligence. 2014. $1-8$.

Geem Zong Woo, Kim Joong Hoon, Loganathan Gobichettipalayam Vasudevan. A new heuristic optimization algorithm: harmony search // simulation. 2001. 76, 2. 60-68.

Ghiasi Ramin, Fathnejat Hamed, Torkzadeh Peyman. A three-stage damage detection method for large-scale space structures using forward substructuring approach and enhanced bat optimization algorithm // Engineering with Computers. 2019. 35, 3. 857-874.

Glover Fred, Laguna Manuel. Tabu search // Handbook of combinatorial optimization. 1998. 2093-2229. 
Goli Alireza, Zare Hassan Khademi, Moghaddam Reza Tavakkoli, Sadeghieh Ahmad. An improved artificial intelligence based on gray wolf optimization and cultural algorithm to predict demand for dairy products: a case study // IJIMAI. 2019. 5, 6. 15-22.

Goudarzi Arman, Ahmadi Afshin, Swanson Andrew G, Van Coller John. Non-Convex optimisation of combined environmental economic dispatch through cultural algorithm with the consideration of the physical constraints of generating units and price penalty factors / / SAIEE Africa Research Journal. 2016. 107, 3. 146-166.

Goudarzi Arman, Swanson Andrew G, Tooryan Fatemeh, Ahmadi Afshin. Non-convex optimization of combined enviromental economic dispatch through the third version of the cultural algorithm (CA3) // 2017 IEEE Texas Power and Energy Conference (TPEC). 2017. 1-6.

Gu Wei, Wu Yonggang. Application of multi-objective cultural algorithm in water resources optimization // 2010 Asia-Pacific Power and Energy Engineering Conference. 2010. 1-4.

Guedria Najeh Ben, Hassine Hichem. Efficient Cultural Algorithm for Structural Damage Detection Problem Based on Modal Data // International Conference on Acoustics and Vibration. 2018. 204-217.

Guo Yi-Nan, Liu Dan-Dan, Cheng Jian, Wang Hui. Adaptive cultural algorithm adopting mixed mutation // Dianzi Xuebao(Acta Electronica Sinica). 2011a. 39, 8. 1913-1918.

Guo Yi-Nan, Wang Hui, Cheng Jian. Adaptive immune clonal selection cultural algorithm / / Dianzi Xuebao(Acta Electronica Sinica). 2010. 38, 4. 966-972.

Guo Yi-nan, Cao Yuan-yuan, Lin Yong, Wang Hui. Knowledge migration based multipopulation cultural algorithm // 2009 Fifth International Conference on Natural Computation. 4. 2009. 331-335.

Guo Yi-nan, Chen Meirong, Wang Chun. Multi-objective quantum cultural algorithm and its application in the wireless sensor networks' energy-efficient coverage optimization // International Conference on Intelligent Data Engineering and Automated Learning. 2013a. 161-167.

Guo Yi-nan, Cheng Jian, Cao Yuan-yuan, Lin Yong. A novel multi-population cultural algorithm adopting knowledge migration // Soft computing. 2011b. 15, 5. 897-905.

Guo Yi-nan, Cheng Jian, Lin Yong, Jiang Xingdong. Optimal Design of Passive Power Filters Based on Multi-objective Cultural Algorithms // International Conference on Intelligent Computing. 2008. 235-242.

Guo Yi-nan, Liu Dandan, Cheng Jian. Multi-population cooperative cultural algorithms // International Conference on Intelligent Computing. 2011c. 199-206.

Guo Yi-nan, Liu Dandan, Liu Yun, Chen Meirong. The coverage optimization for wireless sensor networks based on quantum-inspired cultural algorithm // Proceedings of 2013 Chinese Intelligent Automation Conference. 2013b. 87-96.

Guo Yi-nan, Yang Zhen, Wang Chun, Gong Dunwei. Cultural Particle Swarm Optimization Algorithms for Interval Multi-Objective Problems // International Conference in Swarm Intelligence. 2015. 505-512. 
Guo Yi-nan, Yang Zhen, Wang Chun, Gong Dunwei. Cultural particle swarm optimization algorithms for uncertain multi-objective problems with interval parameters // Natural Computing. 2017. 16, 4. 527-548.

Guo Yi-nan, Zhang Pei, Cheng Jian, Wang Chun, Gong Dunwei. Interval multi-objective quantum-inspired cultural algorithms // Neural Computing and Applications. 2018. 30, 3. 709-722.

Haikal Amira, El-Hosseni Mostafa. Modified cultural-based genetic algorithm for process optimization // Ain Shams Engineering Journal. 2011. 2, 3-4. 173-182.

Haldar Vivekananda, Chakraborty Niladri. Minimisation of power loss by network reconfiguration and capacitor installation using modified cultural algorithm // International Journal of Swarm Intelligence. 2014. 1, 3. 226-245.

Haldar Vivekananda, Chakraborty Niladri. Power loss minimization by optimal capacitor placement in radial distribution system using modified cultural algorithm // International Transactions on Electrical Energy Systems. 2015. 25, 1. 54-71.

He Jianjia, Xu Fuyuan. Chaotic-search-based cultural algorithm for solving unconstrained optimization problem // Modelling and Simulation in Engineering. 2011. 2011. 13.

He Shan, Wu Q Henry, Saunders JR. Group search optimizer: an optimization algorithm inspired by animal searching behavior // IEEE transactions on evolutionary computation. 2009. 13, 5. 973-990.

Ho Nhu Binh, Tay Joc Cing. GENACE: an efficient cultural algorithm for solving the flexible job-shop problem // Proceedings of the 2004 Congress on Evolutionary Computation (IEEE Cat. No. 04TH8753). 2. 2004. 1759-1766.

Hosseinioun Pejman, Kheirabadi Maryam, Tabbakh Seyed Reza Kamel, Ghaemi Reza. A new energy-aware tasks scheduling approach in fog computing using hybrid metaheuristic algorithm // Journal of Parallel and Distributed Computing. 2020.

Huang Haiyan, Liu Mandan, Gu Xingsheng. A cultural algorithm based on multilayer belief spaces and its application in neural network fault classifier // 2008 7th World Congress on Intelligent Control and Automation. 2008. 3990-3995.

Jafari Malihe, Salajegheh Eysa, Salajegheh Javad. An efficient hybrid of elephant herding optimization and cultural algorithm for optimal design of trusses // Engineering with Computers. 2019. 35, 3. 781-801.

Jalili Shahin, Hosseinzadeh Yousef. A cultural algorithm for optimal design of truss structures / / Latin American Journal of Solids and Structures. 2015. 12, 9. 1721-1747.

Jalili Shahin, Hosseinzadeh Yousef, Rabczuk Timon. Simultaneous Size and Shape Optimization of Dome-Shaped Structures Using Improved Cultural Algorithm / / Sociocultural Inspired Metaheuristics. 2019. 93-120.

Jalili Shahin, Husseinzadeh Kashan Ali. An optics inspired optimization method for optimal design of truss structures / / The Structural Design of Tall and Special Buildings. 2019. 28, 6. e1598. 
Jalili Shahin, Kashan Ali Husseinzadeh, Hosseinzadeh Yousef. League championship algorithms for optimum design of pin-jointed structures // Journal of Computing in Civil Engineering. 2017. 31, 2. 04016048.

Jayyousi Thaer W, Reynolds Robert G. Extracting Urban Occupational Plans Using Cultural Algorithms [Application Notes] / / IEEE Computational Intelligence Magazine. 2014. 9, 3. 66-87.

Jin Xidong, Reynolds Robert G. Using knowledge-based evolutionary computation to solve nonlinear constraint optimization problems: a cultural algorithm approach // Proceedings of the 1999 congress on evolutionary computation-CEC99 (Cat. No. 99TH8406). 3. 1999a. 1672-1678.

Jin Xidong, Reynolds Robert G. Using knowledge-based system with hierarchical architecture to guide the search of evolutionary computation // Proceedings 11th International Conference on Tools with Artificial Intelligence. 1999b. 29-36.

Jin Xidong, Reynolds Robert G. Mining knowledge in large scale databases using cultural algorithms with constraint handling mechanisms // Proceedings of the 2000 Congress on Evolutionary Computation. CEC00 (Cat. No. 00TH8512). 2. 2000. 14981506.

Judeh Thair, Jayyousi Thaer, Acharya Lipi, Reynolds Robert G, Zhu Dongxiao. Gene set cultural algorithm: A cultural algorithm approach to reconstruct networks from gene sets // Proceedings of the International Conference on Bioinformatics, Computational Biology and Biomedical Informatics. 2013. 641-648.

Judeh Thair, Jayyousi Thaer, Acharya Lipi, Reynolds Robert G, Zhu Dongxiao. GSCA: Reconstructing biological pathway topologies using a cultural algorithms approach // 2014 IEEE Congress on Evolutionary Computation (CEC). 2014. 2206-2213.

Jun Ma, ZHANG Jian-pei, Jing YANG, CHENG Li-li. Research on cultural algorithm for solving routing problem of mobile agent // The Journal of China Universities of Posts and Telecommunications. 2008. 15, 4. 121-125.

Karaboga Dervis, Gorkemli Beyza, Ozturk Celal, Karaboga Nurhan. A comprehensive survey: artificial bee colony $(\mathrm{ABC})$ algorithm and applications // Artificial Intelligence Review. 2014. 42, 1. 21-57.

Karakatič Sašo. Optimizing nonlinear charging times of electric vehicle routing with genetic algorithm // Expert Systems with Applications. 2020. 114039.

Kashan Ali Husseinzadeh. League championship algorithm: a new algorithm for numerical function optimization // 2009 international conference of soft computing and pattern recognition. 2009. 43-48.

Kaveh A, Talatahari S. A novel heuristic optimization method: charged system search // Acta Mechanica. 2010. 213, 3-4. 267-289.

Kaveh Ali, Mahdavi Vahid Reza. Colliding bodies optimization: a novel meta-heuristic method / / Computers \& Structures. 2014. 139. 18-27. 
Khan Shafqat Ullah, Qureshi Ijaz Mansoor, Zaman F, Shoaib B, Naveed A, Basit A. Correction of faulty sensors in phased array radars using symmetrical sensor failure technique and cultural algorithm with differential evolution // The Scientific World Journal. 2014. 2014.

Khan Shafqat Ullah, Qureshi Ijaz Mansoor, Zaman Fawad, Khan Wasim. Detecting faulty sensors in an array using symmetrical structure and cultural algorithm hybridized with differential evolution / / Frontiers of Information Technology \& Electronic Engineering. 2017. 18, 2. 235-245.

Khodabakhshian Amin, Hemmati Reza. Multi-machine power system stabilizer design by using cultural algorithms // International Journal of Electrical Power \& Energy Systems. 2013. 44, 1. 571-580.

Kim Yongjun, Cho Sung-Bae. A hybrid cultural algorithm with local search for traveling salesman problem // 2009 IEEE International Symposium on Computational Intelligence in Robotics and Automation-(CIRA). 2009. 188-192.

King Irwin, Li Yangmin, Duan Haibin, Reynolds Robert, Ali Mostafa. Embedding a social fabric component into cultural algorithms toolkit for an enhanced knowledge-driven engineering optimization / / International Journal of Intelligent Computing and Cybernetics. 2008.

Kobti Ziad, Reynolds Robert G, Kohler Tim A. The emergence of social network hierarchy using cultural algorithms // International Journal on Artificial Intelligence Tools. 2006a. 15, 06. 963-978.

Kobti Ziad, Snowdon Anne W, Rahaman Shamual, Dunlop Tina, Kent Robert D. A cultural algorithm to guide driver learning in applying child vehicle safety restraint // 2006 IEEE International Conference on Evolutionary Computation. 2006b. 1111-1118.

Kobti Ziad, others . A multiagent system to solve JSSP using a multi-population cultural algorithm / / Canadian Conference on Artificial Intelligence. 2012. 362-367.

Kobti Ziad, others. Heterogeneous multi-population cultural algorithm // 2013 IEEE Congress on Evolutionary Computation. 2013. 292-299.

Kobti Ziad, others . Heterogeneous multi-population cultural algorithm with a dynamic dimension decomposition strategy // Canadian Conference on Artificial Intelligence. 2014. 345-350.

Kulkarni Vaishali Raghavendra, Desai Veena. Sensor Localization in Wireless Sensor Networks Using Cultural Algorithm // International Journal of Swarm Intelligence Research (IJSIR). 2020. 11, 4. 106-122.

Lagos Carolina, Crawford Broderick, Cabrera Enrique, Soto Ricardo, Rubio José-Miguel, Paredes Fernando. Comparing evolutionary strategies on a biobjective cultural algorithm // The Scientific World Journal. 2014. 2014.

Laland Kevin N. Exploring gene-culture interactions: insights from handedness, sexual selection and niche-construction case studies // Philosophical Transactions of the Royal Society B: Biological Sciences. 2008. 363, 1509. 3577-3589. 
Li Ni, Yi Wenqing, Gong Guanghong. Multi-aircraft cooperative target allocation in BVR air combat using cultural-genetic algorithm / / International Computer Science Conference. 2012. 414-422.

Li Xia, Liu Kunqi, Ma Lixiao, Li Huanzhe. A concurrent-hybrid evolutionary algorithms with multi-child differential evolution and Guotao algorithm based on cultural algorithm framework // International Symposium on Intelligence Computation and Applications. 2010. 123-133.

Liang Xiao Joyce, Guler S Ilgin, Gayah Vikash V. A heuristic method to optimize generic signal phasing and timing plans at signalized intersections using Connected Vehicle technology // Transportation Research Part C: Emerging Technologies. 2020. 111. $156-170$.

Lin Cheng-Jian, Chen Cheng-Hung, Lin Chin-Teng. A hybrid of cooperative particle swarm optimization and cultural algorithm for neural fuzzy networks and its prediction applications / / IEEE Transactions on Systems, Man, and Cybernetics, Part C (Applications and Reviews). 2008. 39, 1. 55-68.

Lin Cheng-Jian, Weng Chia-Chun, Lee Chin-Ling, Lee Chi-Yung. Using an efficient hybrid of cooperative particle swarm optimization and cultural algorithm for neural fuzzy network design // 2009 International Conference on Machine Learning and Cybernetics. 5. 2009. 3076-3082.

Liu Wan-Yu, Lin Chun-Cheng. Spatial forest resource planning using a cultural algorithm with problem-specific information // Environmental Modelling \& Software. 2015. 71. 126-137.

Liu Yan, Yang Zhaosheng, Han Xiufeng. The Research on Cultural Algorithm of Shortest Path in the Digital Map // Green Communications and Networks. 2012. 843-850.

Lu Youlin, Zhou Jianzhong, Qin Hui, Wang Ying, Zhang Yongchuan. A hybrid multiobjective cultural algorithm for short-term environmental/economic hydrothermal scheduling // Energy Conversion and Management. 2011. 52, 5. 2121-2134.

Lynn Nandar, Ali Mostafa Z, Suganthan Ponnuthurai Nagaratnam. Population topologies for particle swarm optimization and differential evolution / / Swarm and evolutionary computation. 2018. 39. 24-35.

Maldonado Fernando, Ochoa Alberto, Arreola Julio, Azpeitia Daniel, Torre Ariel De la, Canales Diego, González Saúl. Intelligent Application to Reduce Transit Accidents in a City Using Cultural Algorithms // Distributed Computing and Artificial Intelligence. 2013. 9-17.

Mao Zhengyan, Xiang Yue, Zhang Yijie, Liu Mandan. A Novel Multi-objective Cultural Algorithm Embedding Five-Element Cycle Optimization / / 2020 IEEE Congress on Evolutionary Computation (CEC). 2020. 1-10.

Massa Werner. Crystal structure determination. 2013.

Meng Wenyue, Wei Chen, Tang Jie. Cultural algorithm based on particle swarm optimization for clearance of flight control law // 2011 6th IEEE Conference on Industrial Electronics and Applications. 2011. 418-423. 
Mohammadhosseini Mahdieh, Haghighat Abolfazl Toroghi, Mahdipour Ebrahim. An efficient energy-aware method for virtual machine placement in cloud data centers using the cultural algorithm / / The Journal of Supercomputing. 2019. 75, 10. 6904-6933.

Mojab Seyed Ziae Mousavi, Ebrahimi Mahdi, Reynolds Robert, Lu Shiyong. iCATS: Scheduling big data workflows in the cloud using cultural algorithms // 2019 IEEE Fifth International Conference on Big Data Computing Service and Applications (BigDataService). 2019. 99-106.

Muhamediyeva DT. Fuzzy cultural algorithm for solving optimization problems // Journal of Physics: Conference Series. 1441, 1. 2020. 012152.

Genetic algorithms. // . 1997.

Murugadass Gopal, Sivakumar Poruran. A hybrid elephant herding optimization and cultural algorithm for energy-balanced cluster head selection scheme to extend the lifetime in WSNs // International Journal of Communication Systems. 2020. 33, 15. e4538.

Nallaperuma Samadhi, Jalili Shahin, Keedwell Edward, Dawn Alex, Oakes-Ash Laurence. Optimisation of Signal Timings in a Road Network // Complex Networks XI. 2020. 257-268.

Nguyen Trung Thanh, Yao Xin. Hybridizing cultural algorithms and local search // International Conference on Intelligent Data Engineering and Automated Learning. 2006. 586-594.

Nguyen Trung Thanh, Yao Xin. An experimental study of hybridizing cultural algorithms and local search / / International Journal of Neural Systems. 2008. 18, 01. 1-17.

Niu Qun, Zeng Tingting, Zhou Zhuo. A novel cultural algorithm based on differential evolution for hybrid flow shop scheduling problems with fuzzy processing time // International Symposium on Integrated Uncertainty in Knowledge Modelling and Decision Making. 2011. 121-132.

Ochoa Alberto, García Yazmani, Yañez Javier, Teymanoglu Yaddik. Using cultural algorithms to improve intelligent logistics // International Conference on Hybrid Artificial Intelligence Systems. 2010. 127-134.

Ochoa Alberto, Trejo Erick, Azpeitia Daniel, Esquinca Néstor, Jaramillo Rubén, Sánchez Jöns, González Saúl, Hernández Arturo. Mobile Device to Measure Ubiquitous Danger in a Great City Based on Cultural Algorithms // Distributed Computing and Artificial Intelligence. 2012. 647-655.

Oloruntoba Oluwabunmi, Cosma Georgina, Liotta Antonio. Clan-Based Cultural Algorithm for Feature Selection // 2019 International Conference on Data Mining Workshops (ICDMW). 2019. 465-472.

Ostrowski David A, Reynolds Robert G. Knowledge-based software testing agent using evolutionary learning with cultural algorithms // Proceedings of the 1999 Congress on Evolutionary Computation-CEC99 (Cat. No. 99TH8406). 3. 1999. 1657-1663.

Ostrowski David A, Reynolds Robert G. Using Software Engineering Knowledge to Drive Genetic Program Design Using Cultural Algorithms // Genetic Programming Theory and Practice. 2003. 63-80. 
Ostrowski David A, Reynolds Robert G. Using cultural algorithms to evolve strategies for recessionary markets / / Proceedings of the 2004 Congress on Evolutionary Computation (IEEE Cat. No. 04TH8753). 2. 2004. 1780-1785.

Ostrowski David A, Reynolds Robert G. Using Cultural Algorithms To Evolve Strategies in A Complex Agent-Based System // Knowledge Incorporation in Evolutionary Computation. 2005. 81-102.

Pan Zhong-Liang, Chen Ling, Zhang Guang-Zhao. Cultural algorithm for minimization of binary decision diagram and its application in crosstalk fault detection // International Journal of Automation and Computing. 2010. 7, 1. 70-77.

Pan Zhong-Liang, Chen Ling, Zhang Guang-Zhao. Efficient design method for cell allocation in hybrid CMOS/nanodevices using a cultural algorithm with chaotic behavior // Frontiers of Physics. 2016. 11, 2. 116201.

Peng Bin, Reynolds Robert G. Cultural algorithms: Knowledge learning in dynamic environments // Proceedings of the 2004 Congress on Evolutionary Computation (IEEE Cat. No. 04TH8753). 2. 2004. 1751-1758.

Perera Ricardo, Ruiz Antonio. A multistage FE updating procedure for damage identification in large-scale structures based on multiobjective evolutionary optimization // Mechanical Systems and Signal Processing. 2008. 22, 4. 970-991.

Pierezan Juliano, Maidl Gabriel, Yamao Eduardo Massashi, Santos Coelho Leandro dos, Mariani Viviana Cocco. Cultural coyote optimization algorithm applied to a heavy duty gas turbine operation // Energy Conversion and Management. 2019. 199. 111932.

Prestwich Steven David, Tarim SA, Rossi Roberto, Hnich Brahim. A cultural algorithm for pomdps from stochastic inventory control // International Workshop on Hybrid Metaheuristics. 2008. 16-28.

Rao R Venkata, Savsani Vimal J, Vakharia DP. Teaching-learning-based optimization: a novel method for constrained mechanical design optimization problems // Computer-Aided Design. 2011. 43, 3. 303-315.

Rashedi Esmat, Nezamabadi-Pour Hossein, Saryazdi Saeid. GSA: a gravitational search algorithm // Information sciences. 2009. 179, 13. 2232-2248.

Reyes Laura Cruz, Zezzatti Carlos Alberto Ochoa Ortíz, Santillán Claudia Gómez, Hernández Paula Hernández, Fuerte Mercedes Villa. A cultural algorithm for the urban public transportation // International Conference on Hybrid Artificial Intelligence Systems. 2010. 135-142.

Reynolds R. G., Rolnick S. R. Learning the parameters for a gradient-based approach to image segmentation from the results of a region growing approach using cultural algorithms // Proceedings of 1995 IEEE International Conference on Evolutionary Computation. 2. 1995. 819-824 vol.2.

Reynolds Robert, Al-Shehri Hasan. The use of cultural algorithms with evolutionary programming to control the data mining of large-scale spatio-temporal databases // 1997 IEEE International Conference on Systems, Man, and Cybernetics. Computational Cybernetics and Simulation. 5. 1997. 4098-4103. 
Reynolds Robert, Al-Shehri Hasan. The use of cultural algorithms with evolutionary programming to guide decision tree induction in large databases // 1998 IEEE International Conference on Evolutionary Computation Proceedings. IEEE World Congress on Computational Intelligence (Cat. No. 98TH8360). 1998. 541-546.

Reynolds Robert, Nazzal Ayman. Using cultural algorithm with evolutionary computing to extract site location decisions from spatio-temporal databases // International Conference on Evolutionary Programming. 1997. 443-456.

Reynolds Robert G. An introduction to cultural algorithms // Proceedings of the third annual conference on evolutionary programming. 1994. 131-139.

Reynolds Robert G. Cultural algorithms: Theory and applications // New ideas in optimization. 1999. 367-378.

Reynolds Robert G. Culture on the Edge of Chaos: Cultural Algorithms and the Foundations of Social Intelligence. 2018a.

Reynolds Robert G. The cultural algorithm: Culture on the edge of chaos // Culture on the Edge of Chaos. 2018b. 1-11.

Reynolds Robert G, Ali Mostafa. Computing with the social fabric: The evolution of social intelligence within a cultural framework // IEEE computational intelligence magazine. 2008a. 3, 1. 18-30.

Reynolds Robert G, Ali Mostafa, Jayyousi Thaer. Mining the social fabric of archaic urban centers with cultural algorithms // Computer. 2008a. 41, 1. 64-72.

Reynolds Robert G, Ali Mostafa Z. Cultural Algorithms: Knowledge-driven engineering optimization via weaving a social fabric as an enhanced influence function // 2008 IEEE Congress on Evolutionary Computation (IEEE World Congress on Computational Intelligence). 2008b. 4192-4199.

Reynolds Robert G, Ali Mostafa Z. The social fabric approach as an approach to knowledge integration in Cultural Algorithms // 2008 IEEE Congress on Evolutionary Computation (IEEE World Congress on Computational Intelligence). 2008c. 42004207.

Reynolds Robert G, Ali Mostafa Z, Franzel Patrick. Using GP and Cultural Algorithms to Simulate the Evolution of an Ancient Urban Center / / Genetic Programming Theory and Practice V. 2008b. 261-276.

Reynolds Robert G, Chung ChanJin. Fuzzy approaches to acquiring experimental knowledge in cultural algorithms / / Proceedings Ninth IEEE International Conference on Tools with Artificial Intelligence. 1997a. 260-267.

Reynolds Robert G, Chung ChanJin. Knowledge-based self-adaptation in evolutionary programming using cultural algorithms // Proceedings of 1997 IEEE International Conference on Evolutionary Computation (ICEC'97). 1997b. 71-76.

Reynolds Robert G, Chung Chanjin. A cultural algorithm framework to evolve multiagent cooperation with evolutionary programming // International Conference on Evolutionary Programming. 1997c. 323-333. 
Reynolds Robert G, Gawasmeh Yousof A. Evolving heterogeneous social fabrics for the solution of real valued optimization problems using cultural algorithms // 2012 IEEE Congress on Evolutionary Computation. 2012. 1-8.

Reynolds Robert G, Gawasmeh Yousof A, Salaymeh Areej. The Impact of Subcultures in Cultural Algorithm Problem Solving / / 2015 IEEE Symposium Series on Computational Intelligence. 2015. 1876-1884.

Reynolds Robert G, Kinnaird-Heether Leonard. Survival of the fastest: Using cultural algorithms to optimize the design of a controller for a 3D racing game / Computational \& Mathematical Organization Theory. 2003. 9. 227-254.

Reynolds Robert G, Kinnaird-Heether Leonard. Optimization problem solving with auctions in Cultural Algorithms // Memetic Computing. 2013. 5, 2. 83-94.

Reynolds Robert G, Kinnaird-Heether Leonard. Population mechanics and cultural algorithms in the development of a cultural engine // 2017 IEEE Symposium Series on Computational Intelligence (SSCI). 2017a. 1-8.

Reynolds Robert G, Kinnaird-Heether Leonard. Problem solving using social networks in Cultural Algorithms with auctions / / 2017 IEEE Congress on Evolutionary Computation (CEC). 2017b. 2714-2721.

Reynolds Robert G, Maletic Jonathan I. LEARNING TO COOPERATE USING CUL TURAL ALGORITHMS // Proceedings of the Third Annual Conference on Evolutionary Programming, 24-26 Feb 94, San Diego, California, USA. 1994. 140.

Reynolds Robert G, Peng Bin. Cultural algorithms: computational modeling of how cultures learn to solve problems: an engineering example / Cybernetics and Systems: An International Journal. 2005. 36, 8. 753-771.

Reynolds Robert G, Peng Bin, Ali Mostafa Z. The role of culture in the emergence of decision-making roles: An example using cultural algorithms // Complexity. 2008c. $13,3.27-42$.

Reynolds Robert G, Rolnick Stefan R. Learning the parameters for a gradient-based approach to image segmentation using cultural algorithms // Proceedings First International Symposium on Intelligence in Neural and Biological Systems. INBS'95. 1995. 240-247.

Reynolds Robert $G$, Saleem SM. The impact of environmental dynamics on cultural emergence / / Perspectives on Adaptions in Natural and Artificial Systems. 2005. 253-280.

Reynolds Robert G, Sverdlik William. Learning the behavior of Boolean circuits from examples using cultural algorithms // Adaptive and Learning Systems II. 1962. 1993. 177-188.

Reynolds Robert G, Sverdlik William. An evolution-based approach to program understanding using cultural algorithms / / International Journal of Software Engineering and Knowledge Engineering. 1995. 5, 02. 211-226.

Reynolds Robert G, Zhu Shinin. Knowledge-based function optimization using fuzzy cultural algorithms with evolutionary programming / / IEEE Transactions on Systems, Man, and Cybernetics, Part B (Cybernetics). 2001. 31, 1. 1-18. 
Reynolds Robert Gene. An adaptive computer model of the evolution of agriculture for hunter-gatherers in the valley of Oaxaca, Mexico. 1979.

Richerson PJ, Boyd R. Not by genes alone: how culture transformed human evolution. Chicago: Univ. 2005.

Rychtyckyi N, Reynolds Robert G. Using cultural algorithms to improve performance in semantic networks // Proceedings of the 1999 Congress on Evolutionary Computation-CEC99 (Cat. No. 99TH8406). 3. 1999. 1651-1656.

Rychtyckyj Nestor, Ostrowski David, Schleis George, Reynolds Robert G. Using cultural algorithms in industry / / Proceedings of the 2003 IEEE Swarm Intelligence Symposium. SIS'03 (Cat. No. 03EX706). 2003. 187-192.

Rychtyckyj Nestor, Reynolds Robert G. Learning to re-engineer semantic networks using cultural algorithms // International Conference on Evolutionary Programming. 1998. 179-190.

Rychtyckyj Nestor, Reynolds Robert G. Assessing the performance of cultural algorithms for semantic network re-engineering / / Proceedings of the 2000 Congress on Evolutionary Computation. CEC00 (Cat. No. 00TH8512). 2. 2000. 1482-1491.

Rychtyckyj Nestor, Reynolds Robert G. Using cultural algorithms to improve knowledge base maintainability // Proceedings of the 2001 Genetic and Evolutionary Computation Conference GECCO-2001. 2001. 1405-1412.

Rychtyckyj Nestor, Reynolds Robert G. Knowledge base maintenance using cultural algorithms: application to the DLMS manufacturing process planning system at Ford Motor Company // Proceedings of the 2002 Congress on Evolutionary Computation. CEC'02 (Cat. No. 02TH8600). 1. 2002. 855-860.

Rychtyckyj Nestor, Reynolds Robert G. Using cultural algorithms to re-engineer largescale semantic networks // International Journal of Software Engineering and Knowledge Engineering. 2005. 15, 04. 665-693.

Saleem Saleh, Reynolds Robert. Cultural algorithms in dynamic environments // Proceedings of the 2000 Congress on Evolutionary Computation. CEC00 (Cat. No. 00TH8512). 2. 2000. 1513-1520.

Samà M., DAriano A., Toli A., Pacciarelli D., Corman F. Metaheuristics for Real-Time NearOptimal Train Scheduling and Routing / / 2015 IEEE 18th International Conference on Intelligent Transportation Systems. 2015. 1678-1683.

Santos Coelho Leandro dos, Alotto Piergiorgio. Electromagnetic optimization using a cultural self-organizing migrating algorithm approach based on normative knowledge / / IEEE Transactions on Magnetics. 2009. 45, 3. 1446-1449.

Santos Coelho Leandro dos, Mariani Viviana Cocco. An efficient particle swarm optimization approach based on cultural algorithm applied to mechanical design // 2006 IEEE International Conference on Evolutionary Computation. 2006. 1099-1104.

Santos Coelho Leandro dos, Souza Rodrigo Clemente Thom, Mariani Viviana Cocco. Improved differential evolution approach based on cultural algorithm and diversity measure applied to solve economic load dispatch problems // Mathematics and Computers in Simulation. 2009. 79, 10. 3136-3147. 
Selvarajah Kalyani, Kobti Ziad, Kargar Mehdi. A Cultural Algorithm for Determining Similarity Values Between Users in Recommender Systems / / International Conference on the Applications of Evolutionary Computation (Part of EvoStar). 2019. 270-283.

Selvarajah Kalyani, Kobti Ziad, Kargar Mehdi. Cultural Algorithms for Cluster Hires in Social Networks // Procedia Computer Science. 2020. 170. 514-521.

Selvarajaha Kalyani, Zadeha Pooya Moradian, Kargarb Mehdi, Kobtia Ziad. Identifying a Team of Experts in Social Networks using a Cultural Algorithm // Procedia Computer Science. 2019. 151. 477-484.

Shah Priyanshi, Kobti Ziad. Multimodal fake news detection using a Cultural Algorithm with situational and normative knowledge // 2020 IEEE Congress on Evolutionary Computation (CEC). 2020. 1-7.

Simon Dan. Biogeography-based optimization // IEEE transactions on evolutionary computation. 2008. 12, 6. 702-713.

Singh Dilpreet, Zadeh Pooya Moradian, Kobti Ziad. A Multilevel Cooperative MultiPopulation Cultural Algorithm / / 2018 Innovations in Intelligent Systems and Applications (INISTA). 2018. 1-5.

Soza Carlos, Becerra Ricardo Landa, Riff María Cristina, Coello Carlos A Coello. Solving timetabling problems using a cultural algorithm // Applied Soft Computing. 2011. $11,1.337-344$.

Soza Carlos, Landa Ricardo, Riff María Cristina, Coello Carlos. A cultural algorithm with operator parameters control for solving timetabling problems / / International Fuzzy Systems Association World Congress. 2007. 810-819.

Srinivasan Sujatha, Muruganandam S. Automatic Pruning of Rules Through Multiobjective Optimization-A Case Study with a Multi-objective Cultural Algorithm // Intelligent Computing and Innovation on Data Science. 2020. 117-125.

Srinivasan Sujatha, Ramakrishnan Sivakumar. Multi objective optimization of classification rules using cultural algorithms // Procedia Engineering. 2012. 30. 457-465.

Srinivasan Sujatha, Ramakrishnan Sivakumar. Rule discovery with a multi objective cultural algorithm / / Advances in Computing and Information Technology. 2013a. 141150.

Srinivasan Sujatha, Ramakrishnan Sivakumar. A social intelligent system for multiobjective optimization of classification rules using cultural algorithms // Computing. 2013b. 95, 4. 327-350.

Stanley Samuel Dustin, Kattan Khalid, Reynolds Robert. CAPSO: A Parallelized Multiobjective Cultural Algorithm Particle Swarm Optimizer // 2019 IEEE Congress on Evolutionary Computation (CEC). 2019. 3060-3069.

Sternberg Michael, Reynolds Robert G. Using cultural algorithms to support reengineering of rule-based expert systems in dynamic performance environments: a case study in fraud detection // IEEE Transactions on Evolutionary Computation. 1997. 1, 4. 225-243. 
Storn Rainer, Price Kenneth. Differential evolution-a simple and efficient heuristic for global optimization over continuous spaces / / Journal of global optimization. 1997. $11,4.341-359$.

Sun Xue-Mei, Lv Xiao-Yu, Duan Xin-Ming. Novel QoS routing algorithm based on cultural-simulated annealing algorithm // 2009 Second International Conference on Intelligent Networks and Intelligent Systems. 2009. 209-212.

Sun Yang, Zhang Lingbo, Gu Xingsheng. Co-evolutionary cultural based particle swarm optimization algorithm // Life System Modeling and Intelligent Computing. 2010. $1-7$.

Sun Yang, Zhang Lingbo, Gu Xingsheng. A hybrid co-evolutionary cultural algorithm based on particle swarm optimization for solving global optimization problems // Neurocomputing. 2012. 98. 76-89.

Tang Wanwan, Li Yanda. Constrained optimization using triple spaces cultured genetic algorithm / / 2008 Fourth International Conference on Natural Computation. 6. 2008. 589-593.

Tavakoli Mohamad Reza, Rasouli Vahid, Nasajpour Hamid Reza, Shaarbafchizadeh Majid. A new simultaneous coordinated design of STATCOM controller and power system stabilizer for power systems using cultural algorithm // 2014 IEEE International Energy Conference (ENERGYCON). 2014. 446-450.

Terán Juan, Aguilar Jose, Cerrada Mariela. Integration in industrial automation based on multi-agent systems using cultural algorithms for optimizing the coordination mechanisms / / Computers in Industry. 2017. 91. 11-23.

Tremayne Maryjane, Chong Samantha Y, Bell Duncan. Optimisation of algorithm control parameters in cultural differential evolution applied to molecular crystallography // Frontiers of Computer Science in China. 2009. 3, 1. 101.

Unold Olgierd, Tarnawski Radosław. Cultural Ant Colony Optimization on GPUs for Travelling Salesman Problem // International Workshop on Machine Learning, Optimization, and Big Data. 2016. 317-329.

Unold Olgierd, Tarnawski Radosław. A Parallel Framework for Multi-Population Cultural Algorithm and Its Applications in TSP / / International Workshop on Machine Learning, Optimization, and Big Data. 2017. 470-482.

Van Laarhoven Peter JM, Aarts Emile HL. Simulated annealing // Simulated annealing: Theory and applications. 1987. 7-15.

Vitale Kevin, O'Shea John, Reynolds Robert G, Che Xiangdong. Learning group behavior in games: Using Cultural Algorithms: The land bridge game engine example // 2011 IEEE Symposium on Swarm Intelligence. 2011a. 1-9.

Vitale Kevin, Reynolds Robert, O'Shea John, Meadows Guy. Exploring ancient landscapes under lake Huron using cultural algorithms // Procedia Computer Science. 2011b. 6. 303-310.

Wang Gai-Ge, Deb Suash, Gao Xiao-Zhi, Coelho Leandro Dos Santos. A new metaheuristic optimisation algorithm motivated by elephant herding behaviour // International Journal of Bio-Inspired Computation. 2016. 8, 6. 394-409. 
Wang Wei, Song Yuling, Xue Yanbing, Jin Hongling, Hou Juncai, Zhao Minglei. An optimal vibration control strategy for a vehicle's active suspension based on improved cultural algorithm / / Applied Soft Computing. 2015. 28. 167-174.

Wang Xingmei, Hao Wenqian, Li Qiming. An adaptive cultural algorithm with improved quantum-behaved particle swarm optimization for sonar image detection // Scientific reports. 2017. 7, 1. 1-16.

Wang Ying, Zhou Jianzhong, Lu Youlin, Qin Hui, Zhang Yongchuan. Chaos cultural particle swarm optimization and its application // International Symposium on Neural Networks. 2009. 30-40.

Waris Faisal, Reynolds Robert G. Using cultural algorithms to improve wearable device gesture recognition performance / / 2015 IEEE Symposium Series on Computational Intelligence. 2015. 625-633.

Waris Faisal, Reynolds Robert G. Optimizing AI Pipelines: A Game-Theoretic Cultural Algorithms Approach / / 2018 IEEE Congress on Evolutionary Computation (CEC). 2018. 1-10.

Wei Zhou, Yan-Ping Bu. Cultural algorithm based on particle swarm optimization for partner selection of virtual enterprise // Proceedings of the 31st Chinese Control Conference. 2012. 2238-2241.

Wu Ying, Gao Xiao-Zhi, Huang Xian-Lin, Zenger Kai. A hybrid optimization method of particle swarm optimization and cultural algorithm // 2010 Sixth International Conference on Natural Computation. 5. 2010. 2515-2519.

Xiao Benxian, Xiao Jun, Dong Xueping, Tao Yunchao, Lu Cheng. Research of PID parameter optimization based-on cultural based Ant Colony Algorithm for superheated steam temperature // Proceedings of the 29th Chinese Control Conference. 2010. 51715176 .

Xie Wei, Ji Chang-ming, Li Xin-wu. Particle swarm optimization based on cultural algorithm for short-term optimal operation of cascade hydropower stations / / 2009 Fifth International Conference on Natural Computation. 3. 2009. 289-293.

$X u$ Wei, Wang Raofen, Zhang Lingbo, Gu Xingsheng. A multi-population cultural algorithm with adaptive diversity preservation and its application in ammonia synthesis process // Neural Computing and Applications. 2012. 21, 6. 1129-1140.

$X u$ Wei, Zhang Lingbo, Gu Xingsheng. A novel cultural algorithm and its application to the constrained optimization in ammonia synthesis // Life System Modeling and Intelligent Computing. 2010. 52-58.

Xue H. Adaptive Cultural Algorithm-Based Cuckoo Search for Time-Dependent Vehicle Routing Problem with Stochastic Customers Using Adaptive Fractional Kalman Speed Prediction // Mathematical Problems in Engineering. 2020. 2020.

Xue Xiaowei, Yao Min, Cheng Ran. A novel selection operator of cultural algorithm // Knowledge Engineering and Management. 2011. 71-77.

Xue Zhengui, Guo Yinan. Improved cultural algorithm based on genetic algorithm // 2007 IEEE International Conference on Integration Technology. 2007. 117-122. 
Ya-Li Wu, Li-qing Xu. An improved cultural particle swarm optimization algorithm based on feedback mechanism for economic load dispatch problems // 2010 International Conference on Computational Aspects of Social Networks. 2010. 319-322.

Yan Xuesong, Gong Wenyin, Wu Qinghua. Contaminant source identification of water distribution networks using cultural algorithm // Concurrency and Computation: Practice and Experience. 2017a. 29, 24. e4230.

Yan Xuesong, Song Tao, Wu Qinghua. An improved cultural algorithm and its application in image matching // Multimedia Tools and Applications. 2017b. 76, 13. 1495114968.

Yan Xuesong, Wu Qinghua, Sheng Victor S. A double weighted Naive Bayes with niching cultural algorithm for multi-label classification // International Journal of Pattern Recognition and Artificial Intelligence. 2016. 30, 06. 1650013.

Yang Yuzhen, Gu Xingsheng. Cultural-based genetic tabu algorithm for multiobjective job shop scheduling // Mathematical Problems in Engineering. 2014. 2014.

Yu Lingli, Cai Zixing, Gao Ping'an, Liu Xiaoying. A spatial orthogonal allocation and heterogeneous cultural hybrid algorithm for multirobot exploration mission planning / / Journal of Control Theory and Applications. 2011. 9, 2. 171-176.

Yuan Xiaohui, Nie Hao, He Li, Li Cailin, Zhang Yongchuan. A cultural algorithm for scheduling of hydro producer in the power market // 2008 Second International Conference on Genetic and Evolutionary Computing. 2008. 364-367.

Yuan Xiaohui, Nie Hao, Yuan Yanbin, Su Anjun, Wang Liang. Hydrothermal systems generation scheduling using cultural algorithm // Journal of Hydroinformatics. 2009. $11,1.65-78$.

Yuan Xiaohui, Yuan Yanbin. Application of cultural algorithm to generation scheduling of hydrothermal systems // Energy Conversion and Management. 2006. 47, 15-16. 2192-2201.

Zadeh Pooya Moradian, Kobti Ziad. A multi-population cultural algorithm for community detection in social networks // Procedia Computer Science. 2015. 52. 342-349.

Zannoni Elena, Reynolds Robert G. Learning to control the program evolution process with cultural algorithms // Evolutionary Computation. 1997. 5, 2. 181-211.

Zhang Rui, Zhou Jianzhong, Mo Li, Ouyang Shuo, Liao Xiang. Economic environmental dispatch using an enhanced multi-objective cultural algorithm // Electric Power Systems Research. 2013. 99. 18-29.

Zhang Yu. Research of web search based on cultural algorithm new framework // Procedia Engineering. 2012. 29. 3641-3645.

Zhang Yu-lin, Zhu Xia. A Cultural Particle Swarm Optimization Algorithm for Flow Shop Scheduling Problem // Electrical, Information Engineering and Mechatronics 2011. 2012. 1575-1583.

Zhou Yongquan, Zhang Shaoling, Luo Qifang, Abdel-Baset Mohamed. CCEO: cultural cognitive evolution optimization algorithm / / Soft Computing. 2019. 23, 23. 12561-12583. 